\title{
Effects of Huang Qi Decoction on Endothelial Dysfunction Induced by Homocysteine
}

\author{
Shuang Chu, ${ }^{1}$ Xiao-dong Mao, ${ }^{1}$ Li Wang, ${ }^{1}$ and Wen Peng ${ }^{1,2}$ \\ ${ }^{1}$ Laboratory of Renal Disease, Putuo Hospital, Shanghai University of Traditional Chinese Medicine, Shanghai, China \\ ${ }^{2}$ Department of Nephrology, Putuo Hospital, Shanghai University of Traditional Chinese Medicine, Shanghai, China \\ Correspondence should be addressed to Wen Peng; pengwen_01@vip.sina.com
}

Received 21 April 2016; Revised 22 July 2016; Accepted 3 August 2016

Academic Editor: Yong C. Boo

Copyright (c) 2016 Shuang Chu et al. This is an open access article distributed under the Creative Commons Attribution License, which permits unrestricted use, distribution, and reproduction in any medium, provided the original work is properly cited.

\begin{abstract}
Vascular endothelial dysfunction can be induced by homocysteine (Hcy) through promoted oxidative stress. Huang Qi decoction (HQD) is a traditional Chinese medical formula and its components possess antioxidant effect. The study herein was therefore designed to investigate the effects of HQD at different dosage on endothelial dysfunction induced by Hcy. Tempol and apocynin were used to investigate whether antioxidant mechanisms were involved. Endothelium-dependent relaxation of rat aortas was investigated by isometric tension recordings. Reactive oxygen species (ROS) in human umbilical vein endothelial cells (HUVECs) was determined by DHE staining. The assessment related to oxidative stress and NO bioavailability was performed by assay kits and western blot. In isometric tension experiment, HQD at the dose of 30 or $100 \mu \mathrm{g} / \mathrm{mL}$, tempol, or apocynin prevented impaired endothelium-dependent relaxation in isolated aortas elicited by Hcy. In cellular experiments, substantial enhancement in NADPH oxidase and ROS generation and reduction in NO bioavailability triggered by Hcy were reversed by pretreatment of HQD at the dose of $100 \mu \mathrm{g} / \mathrm{mL}$, tempol, or apocynin. The results proved that HQD at an appropriate dosage presented favorable effects on endothelial dysfunction initiated by Hcy through antioxidant mechanisms. HQD can act as a potent prescription for the treatment of endothelium related vascular complications.
\end{abstract}

\section{Introduction}

Endothelial dysfunction, featured by an impaired endothelium-dependent vasodilatation, has been considered to be involved in the pathogenesis of diabetes mellitus and related vascular complications [1]. Oxidative stress is one of the risk factors that have been found to contribute to vascular endothelial dysfunction and thereby the development of diabetic vascular syndrome $[2,3]$. Inordinately increased plasma homocysteine (Hcy), a situation named hyperhomocysteinemia (Hhcy), is documented to be associated with vascular endothelial dysfunction by impairing nitric oxide(NO-) mediated endothelium-dependent relaxation of large arteries [4]. Hcy has been suspected to promote oxidative stress in endothelial cells through overgeneration of reactive oxygen species (ROS), which partially stems from nicotinamide adenine dinucleotide phosphate (NADPH) oxidase. Then excessively generated ROS would severely restrict the bioavailability of endothelium derived NO, the important regulator of endothelial function in diabetic vascular complications which caused relaxation of vascular muscle layer [5]. Accordingly, decreasing ROS in endothelial cells offers an appealing strategy to normalize Hcy-induced impaired endothelium-dependent vasomotor response and endothelial dysfunction.

Huang Qi decoction (HQD) is a classic traditional Chinese medical formulation consisting of various medicinal herbs, which is first described thousands of years ago. It is comprised of Astragalus (Huang Qi), Poria (Fu Ling), Trichosanthes (Gua Lou), Ophiopogon (Mai Dong), Schisandra (Wu Wei Zi), licorice (Gan Cao), and Rehmannia (Di Huang). Accumulative evidence has revealed that components of HQD possess antioxidant activities or could improve diabetes related syndrome. Astragalus possesses rich antidiabetic active ingredients such as Astragaloside IV and holds potential for alleviating glucose intolerance and hypertriglyceridemia in DM and improving metabolic syndrome and endothelium dysfunction in fructose-fed rats [6]. 
Wang et al. demonstrate the antioxidant capacity of carboxymethyl $(1 \rightarrow 3)$ - $\beta$-D-glucan sulfate from Poria [7]. Liuwei Di Huang, a traditional Chinese herbal formula containing Rehmannia, is demonstrated to suppress chronic inflammation and oxidative stress in obese rats [8]. Triterpenoid or 5-hydroxymethylfurfural from Schisandra is reported to have favorable antioxidant effects on alcoholic liver oxidative injury $[9,10]$. A report demonstrates that Gua Lou Guizhi decoction exerts neuroprotective effect by enhancing endogenous antioxidant enzymatic activities [11]. Accordingly, HQD has a potential antioxidant capacity to improve endothelial dysfunction. Actually, the active compounds in the constituent herbs of HQD have been reported to exert an influence on the vascular endothelial dysfunction, abnormal vasodilation, or vascular reactivity. Catalpol found in Rehmannia and Ophiopogonin D can block hydrogen peroxide $\left(\mathrm{H}_{2} \mathrm{O}_{2}\right)$ induced injuries or apoptosis and is involved in ROS scavenging in human umbilical vein endothelial cells (HUVECs) $[12,13]$. Formononetin and extracts of Schisandra can evoke vascular relaxation through endotheliumdependent NO pathway [14]. Quercetin from Astragalus can improve vascular responsiveness in blood vessels of diabetic rats [15]. Glycyrrhizic Acid from licorice can protect AGE induced endothelial dysfunction in HUVECs [16]. However, the protective effects of HQD on homocysteine induced endothelial dysfunction and the potential mechanisms have not yet been exactly elucidated. Accordingly, we investigate the effects of HQD on endothelium-dependent aortic vasorelaxation and endothelial ROS generation induced by Hcy, thus determining whether the antioxidant mechanism is involved in the beneficial effects of HQD against Hcyinduced endothelial dysfunction.

\section{Materials and Methods}

2.1. Reagents and Materials. The herbs were provided by Shanghai Huayu Chinese Herbs Co. Ltd. (Shanghai, China). Dihydroethidium (DHE) was purchased from Molecular Probes Inc. (Eugene, USA). Antibodies of p47phox, p67phox, p40phox, p22phox, and gp91phox were from Santa Cruz Biotechnology (Santa Cruz, CA, USA); Ras- 1 and $\beta$-actin were obtained from Cell Signaling Technology (Danvers, MA, USA); eNOS and p-eNOS were provided by Abcam (Cambridge, MA, USA). Goat anti-rabbit secondary antibodies were bought from Wuhan Boster Biotech Co. Ltd. (Wuhan, China). ECL developer was from Millipore (Billerica, MA, USA). BCA protein quantity kits and PVDF membranes were provided by Pierce (Rockford, IL, USA). Phenylephrine (PE), acetylcholine (ACh), Hcy, sodium nitroprusside (SNP), tempol, apocynin, and N-nitro-L-arginine methyl ester (L-NAME) were purchased from Sigma Chemical Co. Ltd. (St. Louis, MO, USA). All reagents available were of high purity.

2.2. Preparation of HQD. HQD was composed of crude herbs including Astragalus, Poria, Trichosanthes root, Ophiopogon, Schisandra, licorice, and Rehmannia with the ratio of $2: 2: 2: 2: 1: 1: 3$ (dry weight). The medicinal herb mixture was extracted three times by four times volume of water, and then the concentration of thick decoction was adjusted to $70 \%$ with ethanol overnight. Then the collected supernatant was dried in a drying oven at $105^{\circ} \mathrm{C}$ for 2 days to obtain dry decoction.

2.3. Identification of the Main Compounds in HQD. To identify the active constituents in HQD, the extract was weighed and dissolved in acetonitrile at a concentration of $100 \mathrm{mg} / \mathrm{mL}$. The solution was analyzed by the LC-MS/MS method. LC-MS/MS was conducted on a Shimadzu 30AD HPLC system connected with an Applied Biosystem Sciex Qtrap 5500 mass spectrometer equipped with an ESI ionization source. According to previous reports on HQD, we determined 9 major components (Schisandrin, Liquiritin, Acteoside, Glycyrrhizic Acid, Astilbin, Astragaloside IV, Ruscogenin, Catalpol, and 3,29-dibenzoyl rarounitriol) in HQD. A Thermo Betasil C18 column $(50 \times 2.1 \mathrm{~mm}, 5.0 \mu \mathrm{m})$ was used for separating Schisandrin, Liquiritin, Acteoside, Glycyrrhizic Acid, Astilbin, Astragaloside IV, and Ruscogenin. A Phenomenex Gemini C18 column $(50 \times 4.6 \mathrm{~mm}, 5.0 \mu \mathrm{m})$ was used for Catalpol. A Thermo Hypersil C18 column $(50 \times$ $2.1 \mathrm{~mm}, 5 \mu \mathrm{m}$ ) was used for 3,29-dibenzoyl rarounitriol. The mobile phase for separation of Schisandrin, Liquiritin, Acteoside, Glycyrrhizic Acid, Astilbin, Astragaloside IV, Ruscogenin, and Catalpol consisted of $0.1 \%$ formic acid in water (A) and $0.1 \%$ formic acid in acetonitrile (B) with gradient elution at the flow rate of $0.6 \mathrm{~mL} / \mathrm{min}$. The mobile phase for separation of 3,29-dibenzoyl rarounitriol was $10 \mathrm{mM}$ ammonium acetate and $0.1 \%$ formic acid in water (A) and methanol (B) with gradient elution at the flow rate of $0.6 \mathrm{~mL} / \mathrm{min}$. The gradient elution program was set as follows: $0-0.1 \mathrm{~min} 5 \% \mathrm{~B}$, 0.1-1.3 min 5-95\% B, 1.3-1.7 min 95\% B, 1.7-1.8 min 95-5\% B, and 1.8-3.0 min 5\% B for Schisandrin, Liquiritin, Acteoside, Glycyrrhizic Acid, Astilbin, and Astragaloside IV; 0-0.1 min $2 \% \mathrm{~B}, 0.1-1.3 \mathrm{~min} 2-98 \% \mathrm{~B}, 1.3-1.7 \mathrm{~min} 98 \% \mathrm{~B}, 1.7-1.8 \mathrm{~min} 98-$ $2 \% \mathrm{~B}$, and $1.8-3.0$ min $2 \% \mathrm{~B}$ for Ruscogenin; $0-0.1 \mathrm{~min} 30 \% \mathrm{~B}$, 0.1-1.3 min 30-95\% B, 1.3-1.7 min 95\% B, 1.7-1.8 min 95-30\% $\mathrm{B}$, and $1.8-3.0 \mathrm{~min} 30 \% \mathrm{~B}$ for Catalpol; 0-0.1 min 5\% B, 0.1$1.3 \min 5-98 \% \mathrm{~B}, 1.3-1.7 \min 98 \% \mathrm{~B}, 1.7-1.8 \mathrm{~min} 98-5 \% \mathrm{~B}$, and 1.8-3.0 min 5\% B for 3,29-dibenzoyl rarounitriol.

For MS/MS analysis, optimized parameters were as follows: curtain gas, gas 1 , and gas 2 were 15,40 , and 30 psi, respectively; source temperature was $550^{\circ} \mathrm{C}$; and spray voltage was $5500 \mathrm{~V}$. The detections were performed in multiple reaction monitoring (MRM) mode, and ion transitions were set at $m / z \quad 433.4 \rightarrow m / z \quad 384.3, \quad m / z \quad 417.4 \rightarrow m / z \quad 255.1, \quad m / z$ $449.3 \rightarrow m / z$ 150.7, $m / z$ 684.5 $\rightarrow m / z$ 527.5, $m / z$ 623.2 $\rightarrow m / z 161$, $m / z$ 821.5 $\rightarrow m / z$ 351.1, $m / z$ 829.4 $\rightarrow m / z$ 783.5, $\mathrm{m} / z$ 431.5 $\rightarrow \mathrm{m} / z$ 287.3, and $m / z \quad 361.4 \rightarrow m / z 169$ for Schisandrin, Liquiritin, Astilbin, 3,29-dibenzoyl rarounitriol, Acteoside, Glycyrrhizic Acid, Astragaloside IV, Ruscogenin, and Catalpol, respectively.

2.4. Ex Vivo Isometric Tension Measurement. Male Wistar rats (180-250 g) were from Slaccas Co. Ltd. (Shanghai, China). The study protocols were reviewed and approved by the Ethics Committee of Putuo Hospital, Shanghai University of Traditional Chinese Medicine. Rats were anesthetized 
with sodium pentobarbital. The thoracic aorta was rapidly removed and placed in cold Krebs solution ( $\mathrm{pH}$ 7.4) of the following composition: $118.0 \mathrm{~g} \mathrm{NaCl}, 4.7 \mathrm{~g} \mathrm{KCl}, 1.2 \mathrm{~g}$ $\mathrm{MgCl}_{2} \cdot 6 \mathrm{H}_{2} \mathrm{O}, 1.2 \mathrm{~g} \mathrm{KH}_{2} \mathrm{PO}_{4}, 25.0 \mathrm{~g} \mathrm{NaHCO}_{3}, 2.5 \mathrm{~g} \mathrm{CaCl}_{2}$, and $11 \mathrm{~g}$ glucose. The vessels were dissected to be devoid of adipose and connective tissues and cut into rings at the length of $3 \mathrm{~mm}$. Endothelium was removed by gently rubbing the intimal space with a toothpick. The aortic rings were suspended by two L-shaped stainless steel wires inserted into the lumen, in a $10 \mathrm{~mL}$ organ chambers filled with Krebs solution, maintained at $37^{\circ} \mathrm{C}$ and oxygenated continuously with $95 \% \mathrm{O}_{2}$ and $5 \% \mathrm{CO}_{2}$. The isometric tension was recorded with a force transducer and Power Lab recording system (ADInstruments Pty Ltd). Rings were equilibrated for $60 \mathrm{~min}$ at $3 \mathrm{~g}$. The bath solution was changed every $20 \mathrm{~min}$ before exposing arteries to $\mathrm{KCl}(60 \mathrm{mM})$. After a further equilibration period of $30 \mathrm{~min}$, the integrity of the endothelium was assessed by determining the ability of ACh $\left(10^{-6} \mathrm{M}\right)$ to induce more than $80 \%$ relaxation of rings precontracted with $\mathrm{PE}\left(3 \times 10^{-7} \mathrm{M}\right)$. The endothelium was considered to be removed when there was less than $10 \%$ relaxation response to $\mathrm{ACh}$.

To assess the effects of HQD on isolated aortic rings with intact endothelium precontracted with PE $\left(3 \times 10^{-7} \mathrm{M}\right)$ and determine the dosage used in the subsequent experiments, HQD at a dosage ranging from 0.1 to $1000 \mu \mathrm{g} / \mathrm{mL}$ was cumulatively added into the organ bath with 5 min interval. The rings with intact endothelium were tested in parallel without drug as the control.

To assess the effect of Hcy $(1 \mathrm{mM}, 1 \mathrm{~h})$ on endotheliumdependent or endothelium-independent relaxation, ACh $\left(10^{-10}-10^{-4} \mathrm{M}\right)$ or SNP $\left(10^{-11}-10^{-5} \mathrm{M}\right)$ was added after PE $(3 \times$ $10^{-7} \mathrm{M}$ ) induced contraction. To investigate the role of ROS in the observed inhibitory effect of Hcy, rings were coincubated with tempol $(100 \mu \mathrm{M})$, apocynin $(100 \mu \mathrm{M})$, and Hcy $(1 \mathrm{mM})$. Furthermore, to determine whether HQD has similar effects with tempol and apocynin, rings were treated with HQD $(10,30$, and $100 \mu \mathrm{g} / \mathrm{mL})$ instead of tempol and apocynin. In addition, to determine whether increasing NO production is involved in the protective effect of HQD, tempol, and apocynin on impaired endothelium-dependent relaxation induced by Hcy, aortic rings were pretreated with L-NAME (100 $\mu \mathrm{M}, 30 \mathrm{~min})$ before PE-induced contraction.

2.5. Isolation of Endothelial Cells. Human umbilical vein endothelial cells (HUVECs) were digested from fresh newborn umbilical cords as previously described [17]. Briefly, perfuse veins of umbilical cords with PBS ( $\mathrm{pH} 7.4,0.2 \mathrm{M}$ ) to discard blood cells. Then fill them with $0.1 \%$ collagenase for $15 \mathrm{~min}$ at $37^{\circ} \mathrm{C}$. Thereafter, collect the digestion and centrifuge at $1000 \mathrm{rpm}$ for $5 \mathrm{~min}$. HUVECs were cultured in basal Dulbecco's modified Eagle's medium (DMEM) supplemented with 20\% fetal bovine serum (FBS, Gibco, USA), endothelial cell growth supplement (ECGS), and antibiotics. HUVECs from passages 3-4 were used in the subsequent experiments.

2.6. Measurement of ROS Generation. ROS generation was evaluated using fluorescence probe DHE according to the protocol. After successive treatment with HQD or antioxidants for $30 \mathrm{~min}$ and Hcy for another $30 \mathrm{~min}$, the supernatant was used for analyzing oxidative stress markers including superoxide dismutase (SOD), malondialdehyde (MDA), lipid-peroxides (LPO), and hydrogen peroxide $\left(\mathrm{H}_{2} \mathrm{O}_{2}\right)$ through assay kits (Nanjing Jiancheng Bioengineering Institute, Nanjing, China). HUVECs were continually incubated with the probe DHE for $30 \mathrm{~min}$ at $37^{\circ} \mathrm{C}$. Then cells were washed with PBS to remove free DHE molecules, fixed with paraformaldehyde $(10 \mu \mathrm{g} / \mathrm{mL})$. Fluorescence was monitored via a fluorescence microscope (Nikon TE2000, Japan).

2.7. Measurement of NO Production and Phosphorylation of eNOS. HUVECs were seeded in 24-well plate until $90 \%$ confluence. The cells were incubated with $\operatorname{HQD}(10,30$, and $100 \mu \mathrm{g} / \mathrm{mL})$, tempol $(100 \mu \mathrm{M})$, and apocynin $(100 \mu \mathrm{M})$ for $30 \mathrm{~min}$, after which Hcy $(1 \mathrm{mM})$ was incubated for another $60 \mathrm{~min}$. Then the supernatants were collected for the determination of extracellular NO level by assay kits (Nanjing Jiancheng Bioengineering Institute, Nanjing, China). The cells were washed with ice-cold PBS and collected for the extraction of total protein with RIPA lysis buffer. The concentration of extracted protein was determined using BCA kits. Then SDS-PAGE was performed with $20 \mu \mathrm{g}$ of protein sample to determine the expression of eNOS and p-eNOS.

2.8. Measurement of NADPH Oxidase Production. HUVECs were seeded in 24 -well plate until $90 \%$ confluence. After treatment with HQD $(10,30$, and $100 \mu \mathrm{g} / \mathrm{mL})$, tempol $(100 \mu \mathrm{M})$, and apocynin $(100 \mu \mathrm{M})$ for $30 \mathrm{~min}$, HUVECs were incubated with Hcy $(1 \mathrm{mM})$ for another $60 \mathrm{~min}$. Then the supernatants were collected for the determination of extracellular NADPH oxidase levels by assay kits (Wuhan ColorfulGene Biological Technology Co., Ltd., Wuhan, China). The extracted proteins from HUVECs underwent western blot analysis to determine the expression of NADPH oxidase subunits including p47phox, p67phox, p40phox, p22phox, gp91phox, and Ras-1.

2.9. Statistical Analysis. Responses to ACh and SNP were represented as $\%$ of the maximal contraction to PE. The concentration of ACh that induced $50 \%$ of the maximal relaxation was regarded as the EC50 which was determined by regression analysis of the log dose-response curves. EC50 was expressed as pD2 (-log EC50). All data were expressed as mean \pm SEM. Statistical analysis was performed via Student's unpaired $t$-test between two groups or One-Way Analysis of Variance (ANOVA) with Tukey's post hoc test among more than two groups (GraphPad Prism 5.0 software, GraphPad Prism software Inc., San Diego, California, USA). A value of $P<0.05$ was judged to be significant.

\section{Results}

3.1. Identification of the Compounds in HQD Extract. Nine main constituents were analyzed using LC-MS/MS method. Except for 3,29-dibenzoyl rarounitriol, all other eight components could be detected in HQD and further quantified. The 
- XIC of -MRM (11 pairs): 417.400/255.100 Da ID: GCG-1 from sample 10 (mix-STD-100 ng/mL) of HQT-20160707-1.wiff (turbo spray). Max. $4.7 e 5 \mathrm{cps}$

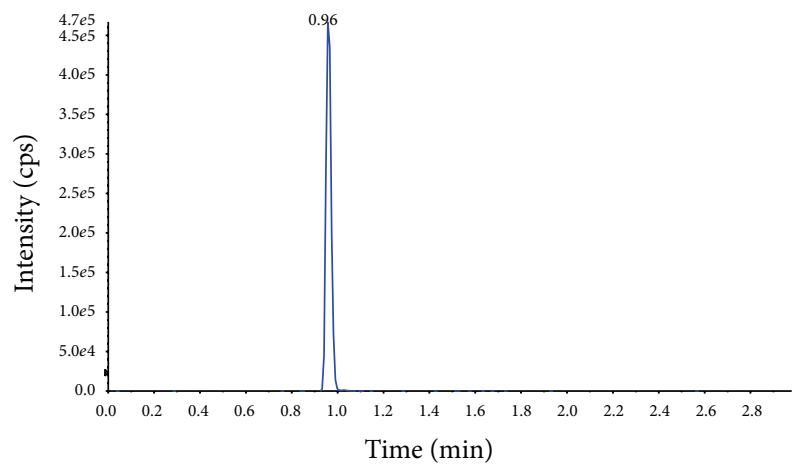

(A)

- XIC of -MRM (11 pairs): 449.300/150.700 Da ID: LXFG-1 from sample 10 (mix-STD-100 ng/mL) of HQT-20160707-1.wiff (turbo spray). Max. 1.2e5 cps

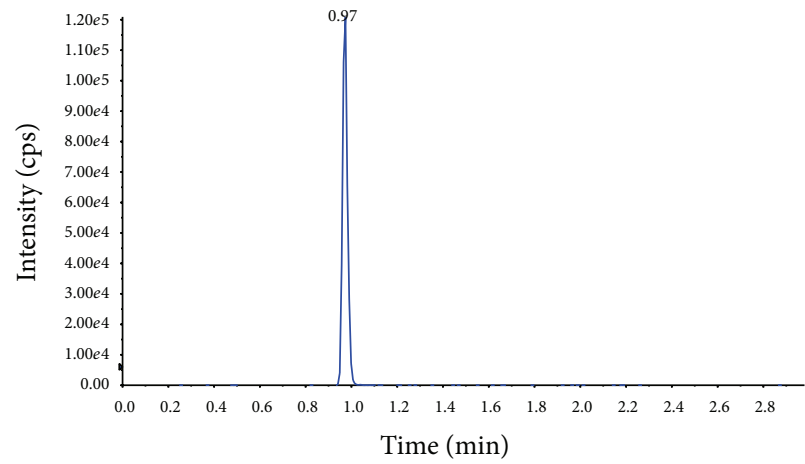

(B)

- XIC of -MRM (11 pairs): 623.200/161.000 Da ID: MRHTG-1 from sample 10 (mix-STD-100 ng/mL)

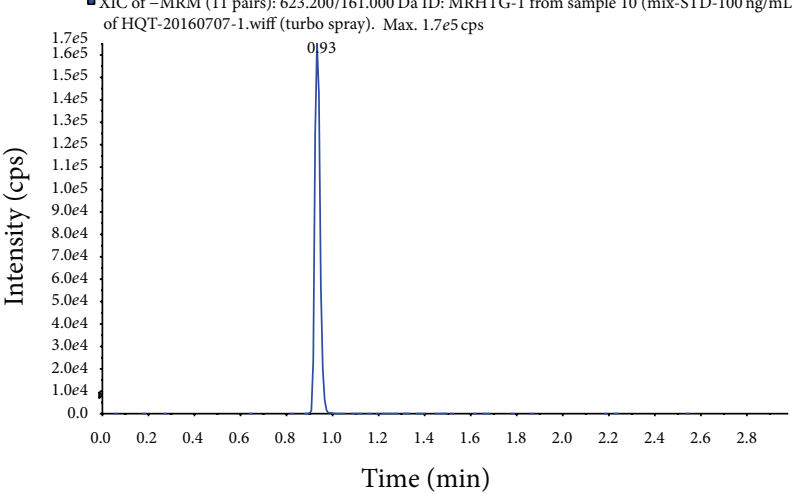

(C)

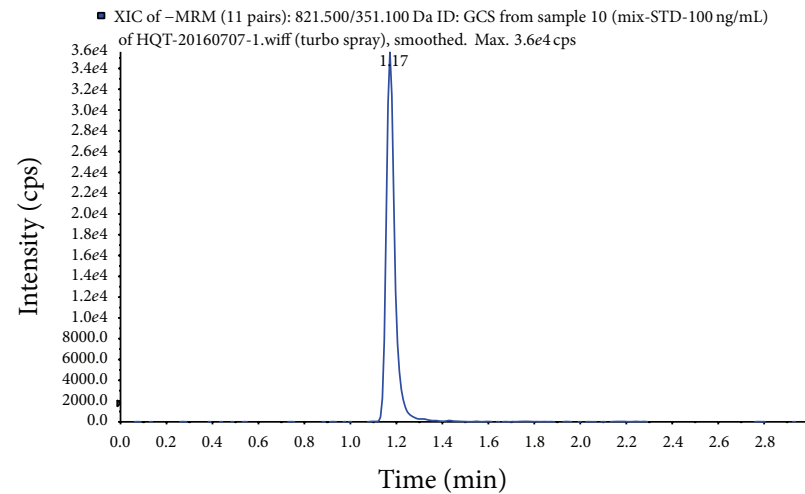

(D)

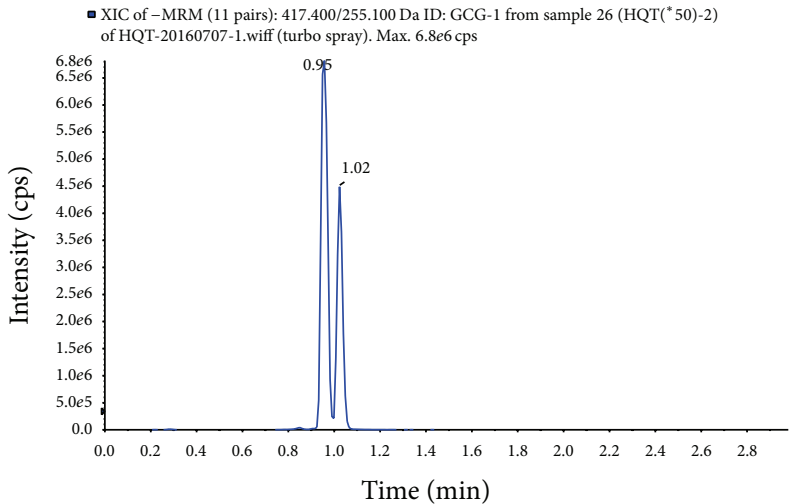

(A)

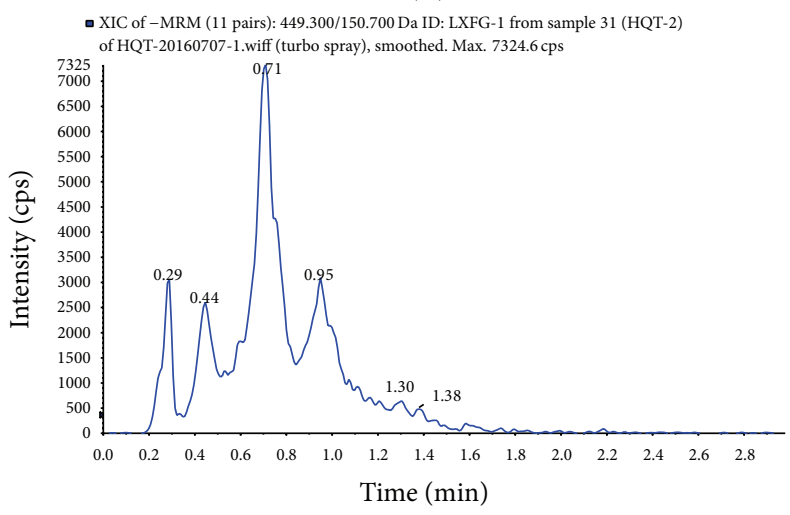

(B)

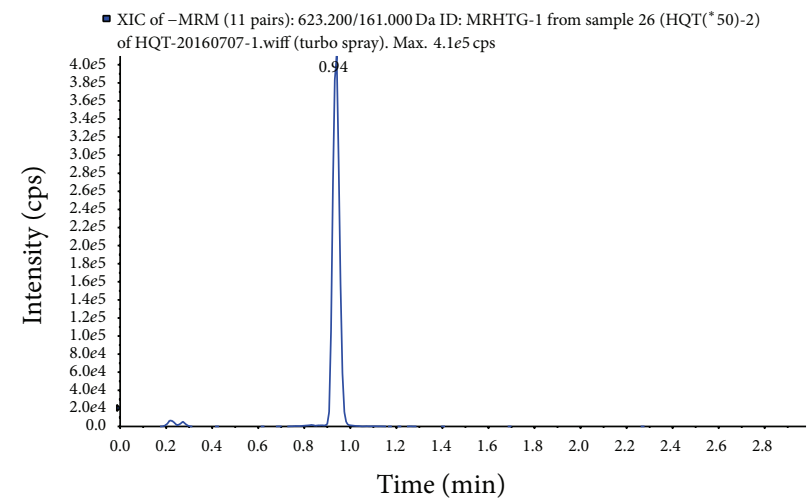

(C)

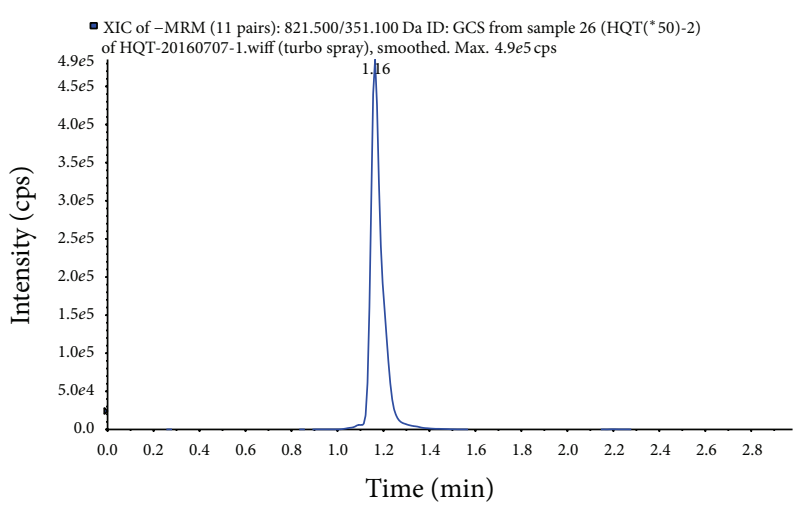

(D)

FIgURE 1: Continued. 


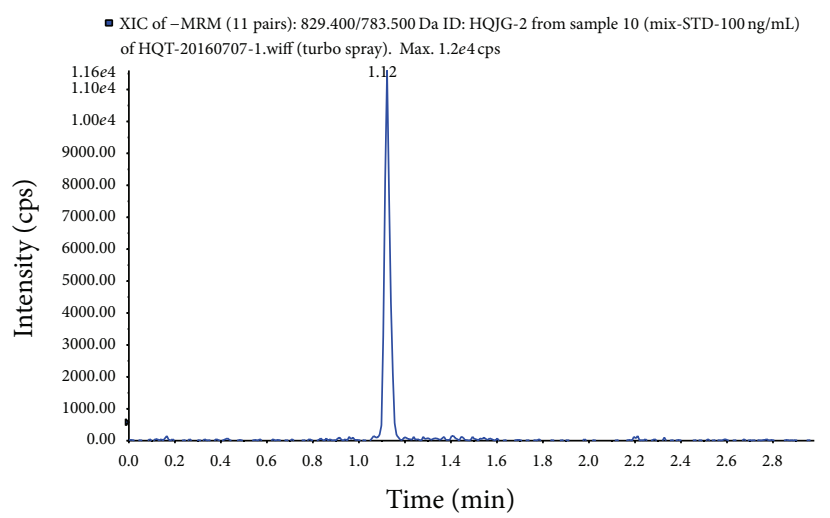

(E)

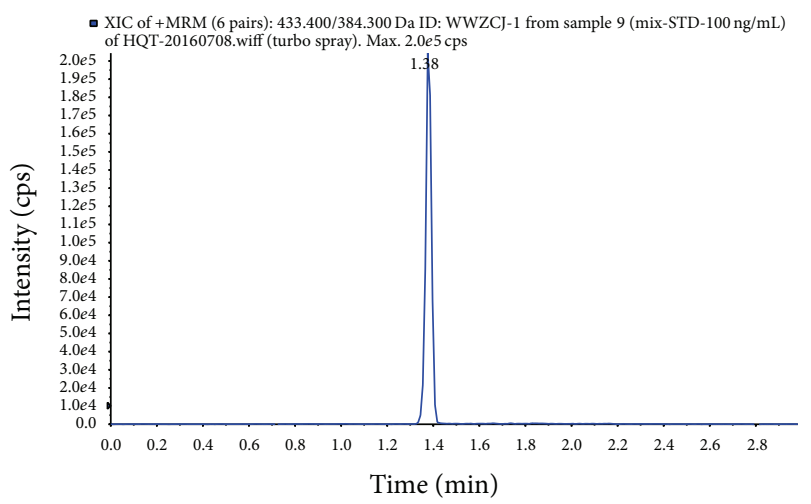

(F)

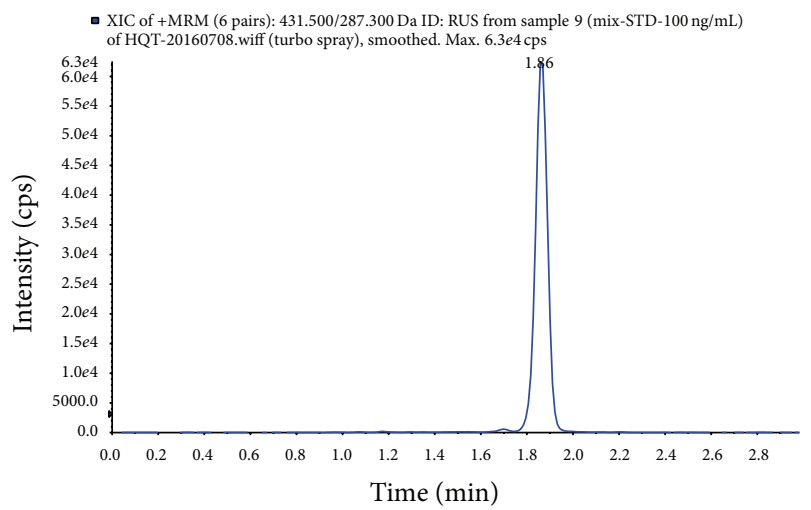

(G)

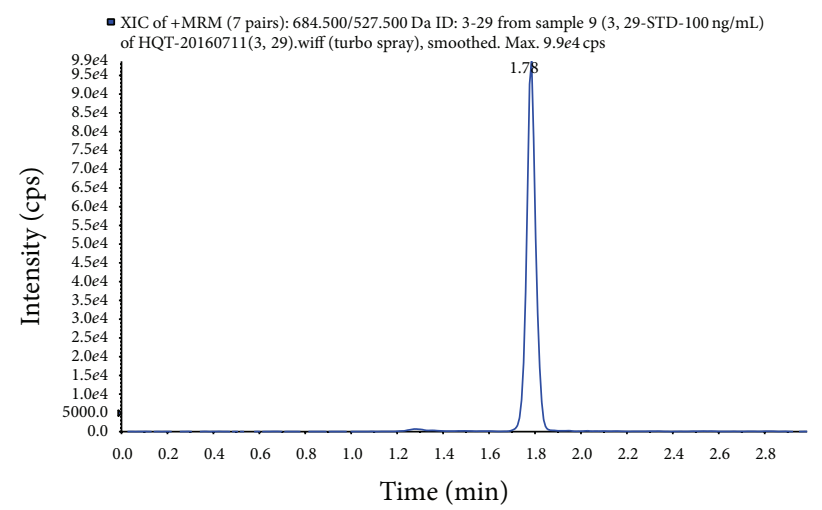

$(\mathrm{H})$

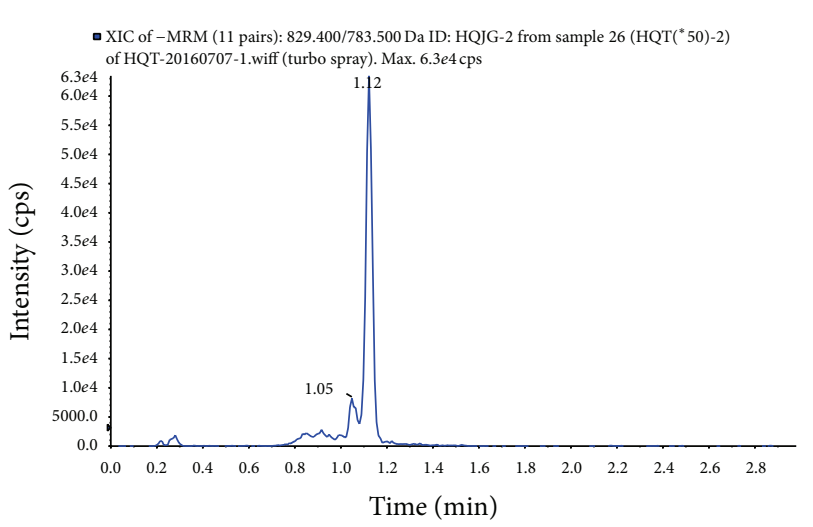

(E)

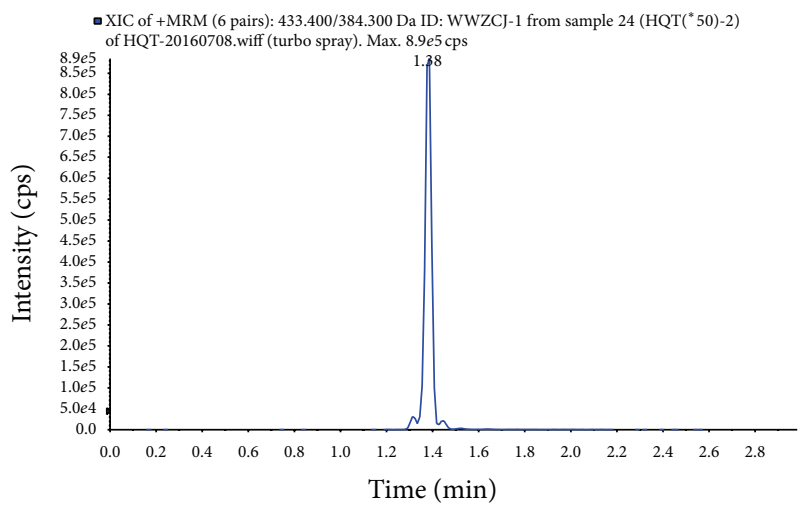

(F)

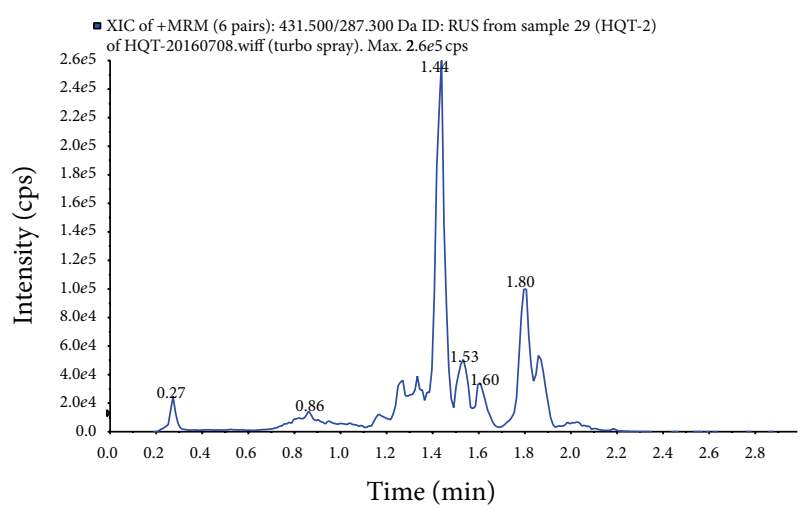

(G)

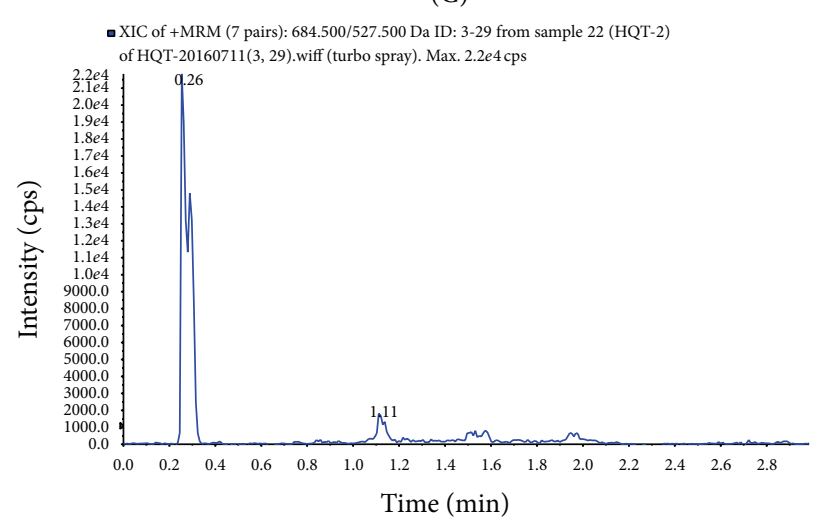

$(\mathrm{H})$

Figure 1: Continued. 


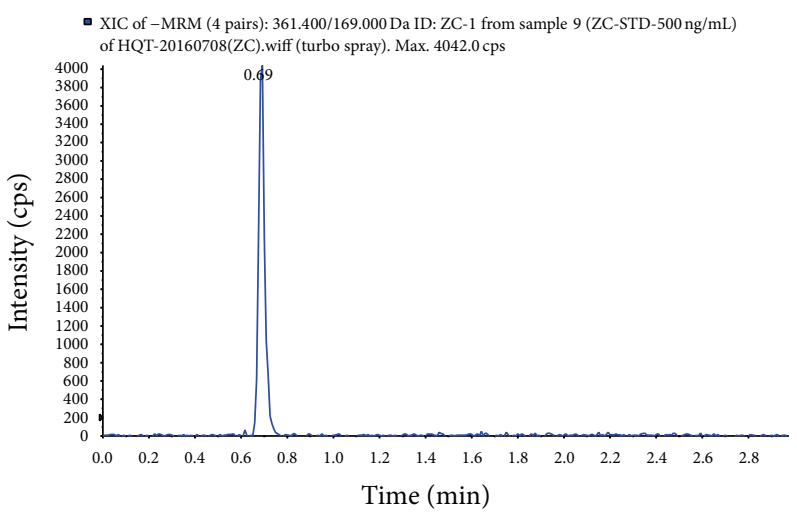

(I)

(a)

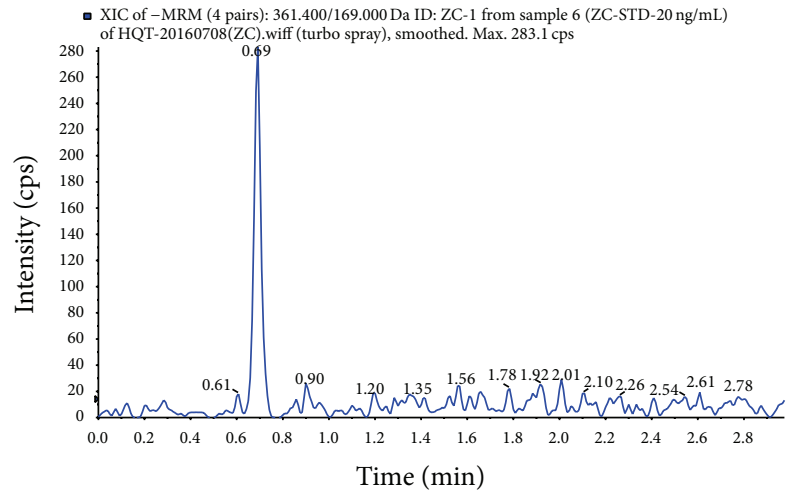

(I)

(b)

FIGURE 1: The extracted ion chromatograms of the detected components in HQD. Reference standard (a) and sample (b). (A) Liquiritin, (B) Astilbin, (C) Acteoside, (D) Glycyrrhizic Acid, (E) Astragaloside IV, (F) Schisandrin, (G) Ruscogenin, (H) 3,29-dibenzoyl rarounitriol, and (I) Catalpol.

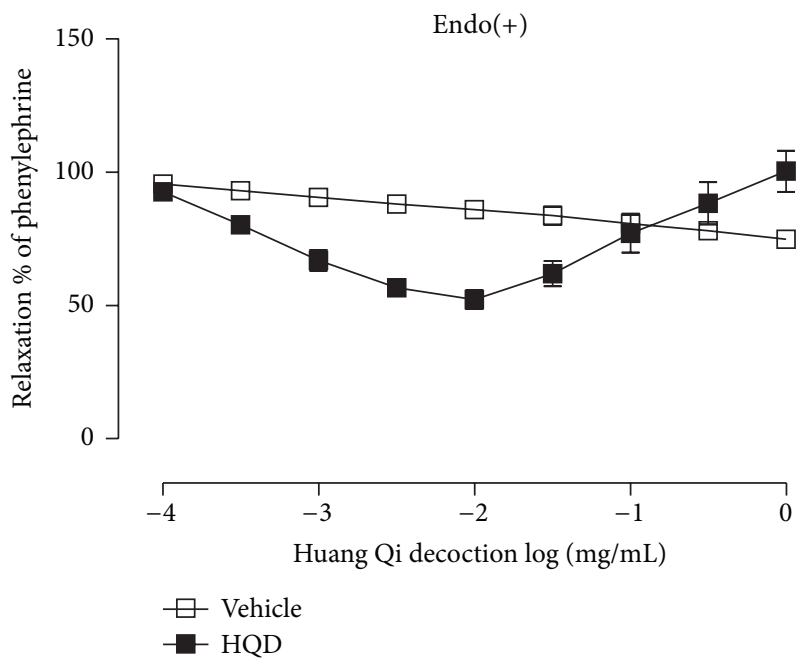

FIGURE 2: Effects of Huang Qi decoction on phenylephrine-induced contractions in aortic rings with endothelium.

ion chromatograms of these compounds were presented in Figure 1. The content of Liquiritin, Astilbin, Acteoside, Glycyrrhizic Acid, Astragaloside IV, Schisandrin, Ruscogenin, and Catalpol in HQD extract was 1047.69, 0.04, 150.46, 879, $337.82,264.69,0.78$, and $0.32 \mu \mathrm{g} / \mathrm{g}$, respectively.

3.2. Effect of HQD on Vasomotor Impairment Induced by Hcy. As shown in Figure 2, in PE-precontracted endotheliumintact rings, low doses of HQD showed relaxation while high dose larger than $100 \mu \mathrm{g} / \mathrm{mL}$ might induce contraction. In Figure 3 , in any group, no difference in relaxation responses to ACh $\left(10^{-6} \mathrm{M}\right)$ in aortic rings was observed at the initial experiment. Compared with the control group $\left(E_{\max }: 95.19 \pm\right.$ $0.97 \%$ and pD2: $7.46 \pm 0.10$ ), pretreatment with Hcy for 60 min reduced ACh-induced relaxation according to the data that $E_{\max }$ fell to $64.15 \pm 5.11 \%$, with a reduction of $\mathrm{pD} 2$

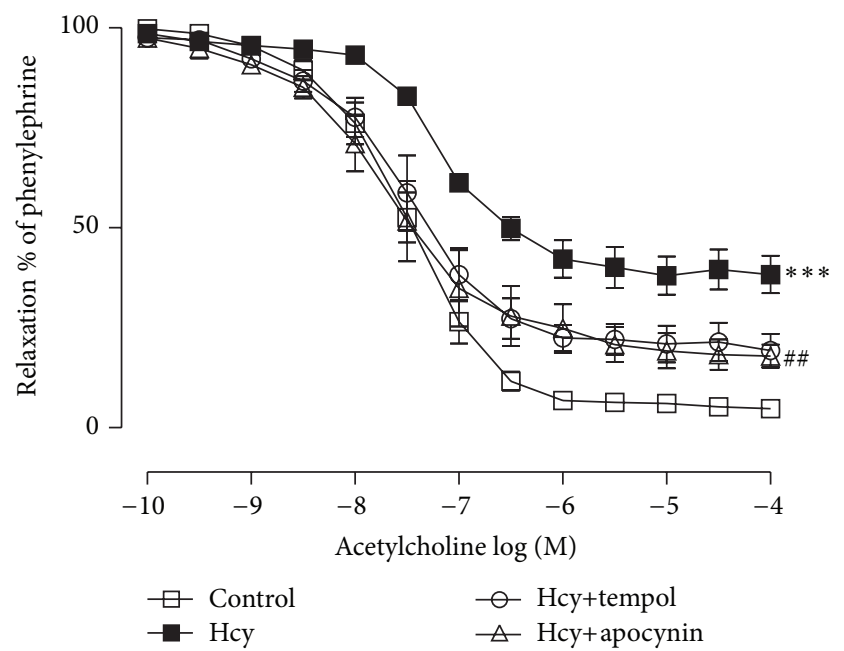

FIGURE 3: Inhibitory effect of homocysteine (Hcy) on the endothelium-dependent relaxation and improvement caused by tempol and apocynin in aortic rings. Values are means \pm SEM $(n=6) .{ }^{* * *} P<$ 0.001 compared with the control group and ${ }^{\# \#} P<0.01$ compared with the Hcy group.

(7.12 \pm 0.08$)$. Endothelium-independent relaxation to SNP was unaffected by Hcy (data not shown).

Cotreatment of HQD (30 and $100 \mu \mathrm{g} / \mathrm{mL})$ with Hcy was able to improve the inhibition of endothelium-dependent relaxation induced by Hcy. $E_{\max }$ and $\mathrm{pD} 2$ were shown as $85.89 \pm 2.20 \%$ or $82.81 \pm 2.51 \%$ versus $64.15 \pm 5.11 \%(P<$ $0.01)$ and $7.63 \pm 0.16$ or $7.59 \pm 0.05$ versus $7.12 \pm 0.08(P<$ 0.05 or $P<0.01$ ) (Figure 4 ).

To confirm the role of ROS in the observed inhibitory effect on endothelium-dependent relaxation by Hcy, tempol and apocynin were used. In Figure 2, $E_{\max }$ and $\mathrm{pD} 2$ were shown as $81.87 \pm 2.05 \%$ or $84.80 \pm 1.62 \%$ versus $64.15 \pm 5.11 \%$ $(P<0.01)$ and $7.49 \pm 0.17$ or $7.56 \pm 0.20$ versus $7.12 \pm 0.08$. 


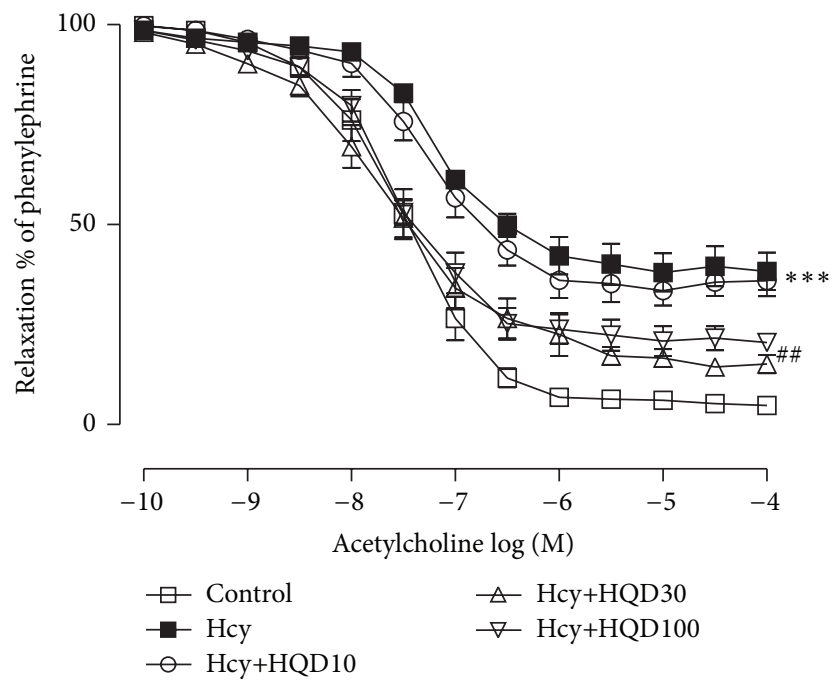

FIgURE 4: Improvement caused by Huang Qi decoction on homocysteine- (Hcy-) induced endothelium-dependent vasorelaxation impairment in aortic rings. Values are means \pm SEM $(n=6)$. ${ }^{* * * *} P<0.001$ compared with the control group and ${ }^{\# \#} P<0.01$ compared with the Hcy group.

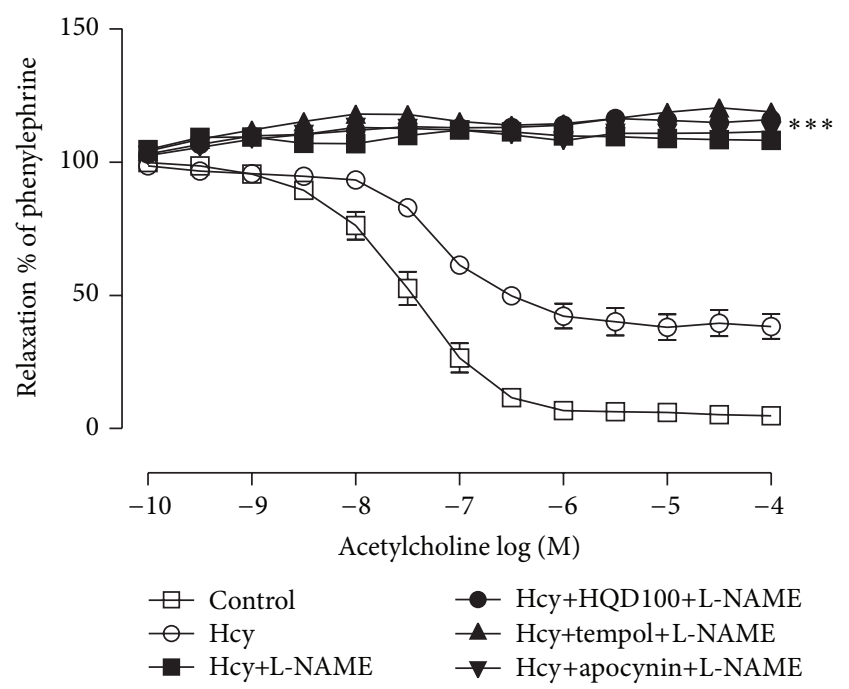

FIGURE 5: Effect of N-nitro-L-arginine methyl ester (L-NAME) on the protection of Huang Qi decoction (HQD), tempol, and apocynin against the impairment of endothelium-dependent relaxation induced by homocysteine (Hcy). Values are means \pm SEM $(n=6)$. ${ }^{* * *} P<0.001$ compared with the Hcy group.

As presented in Figure 5, incubation of aortic rings with L-NAME $(100 \mu \mathrm{M}, 30 \mathrm{~min})$ abolished the improvement of HQD $(100 \mu \mathrm{g} / \mathrm{mL})$, tempol $(100 \mu \mathrm{M})$, and apocynin $(100 \mu \mathrm{M})$ on endothelium-dependent relaxation.

3.3. Effect of $H Q D$ on Intracellular ROS Stimulated by Hcy. Effect of HQD on ROS provocation was determined by DHE fluorescence staining method in HUVECs. As presented in Figures 6(a) and 6(b), Hcy substantially increased DHE fluorescent density, whose effect was eliminated by the treatment of tempol or apocynin. Hcy-provoked ROS production was significantly inhibited by $28 \%$ with the pretreatment of HQD of $100 \mu \mathrm{g} / \mathrm{mL}$ compared with Hcy group. HQD at the dose of 10 or $30 \mu \mathrm{g} / \mathrm{mL}$ did not significantly influence DHE fluorescent intensity. There was not any significant difference in the level of MDA between all the seven groups. The SOD activity and content of $\mathrm{H}_{2} \mathrm{O}_{2}$ in Hcy group were significantly decreased compared with the control group and such decrease was reversed by treatment with HQD, tempol, and apocynin. LPO content was markedly increased in the Hcy group, which was decreased by tempol and apocynin but not by HQD (Figures 6(c)-6(f)).

3.4. Effect of $H Q D$ on $N O$ Production. As expected in Figure $7(\mathrm{a})$, the addition of Hcy significantly reduced NO production by $71 \%$ in comparison with the control group, which was considerably reversed by the pretreatment of tempol or apocynin. The application of HQD at the dose of $100 \mu \mathrm{g} / \mathrm{mL}$ significantly attenuated the decrease of NO level induced by Hcy. However, HQD at the dose of 10 or $30 \mu \mathrm{g} / \mathrm{mL}$ exerted nonsignificant effects on Hcy-induced reduction of NO. Figure 7(b) presented that HQD did not influence phosphorylation of eNOS.

3.5. Effect of HQD on NADPH Oxidase Production. Western blot monitored the expression levels of NADPH oxidase subunits including Ras-1, p47phox, p67phox, p40phox, p22phox, and gp91phox. As shown in Figure 8, HUVECs treated with Hcy alone presented the highest expression level of Ras1, p47phox, p67phox, p40phox, p22phox, and gp91phox, respectively, which could be decreased by tempol or apocynin treatment. Compared with Hcy group, HQD inhibited the expression of NADPH oxidase subunits. In terms of inhibitory efficacy of $\mathrm{HQD}$ at different dosage, HQD at the dose of $100 \mu \mathrm{g} / \mathrm{mL}$ exhibited the best performance.

\section{Discussion}

HQD was a classic traditional Chinese medical formula, whose components had been demonstrated to exert effective effects on diabetic complications through antioxidative mechanism $[6,18]$. In our study, we demonstrated that HQD at an appropriate dosage had potential to treat diabetic vascular complications by improving impaired vasodilation and endothelial function through attenuating oxidative damage. It decreased the expression of NADPH oxidase, inhibited the production of ROS, and increased production of NO, thereby promoting vascular endothelial function of aortic rings.

Oxidative damage was one of the pivotal contributors to diabetic vascular complications and endothelial dysfunction [19]. Previous investigations had addressed the fact that elevated Hcy concentrations would cause vascular endothelium damage via overgenerating oxygen-derived free radicals, in a sense, namely, ROS, which were formed via oxidation of the sulfhydryl base group in Hcy [20, 21]. In the present study, we used Hcy at a concentration of $1 \mathrm{mM}$, which represented approximately 10 -fold dose of clinical plasma levels of 

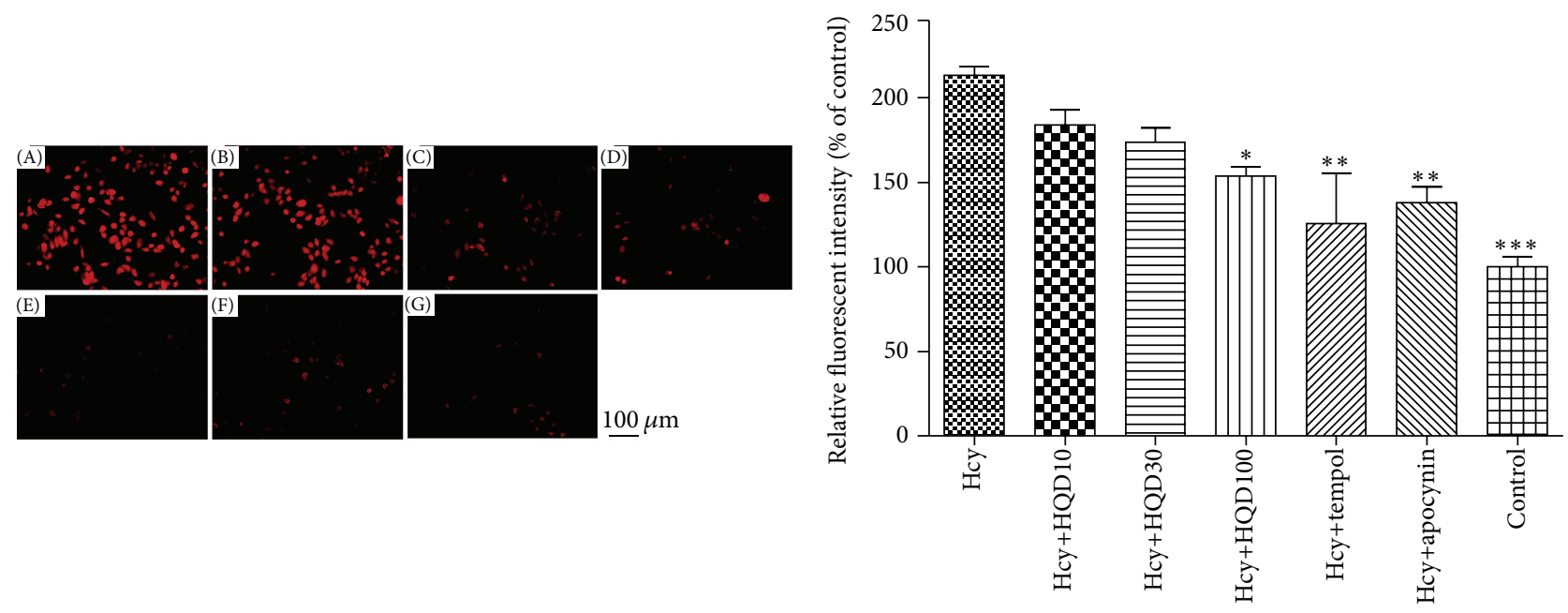

(a)

(b)
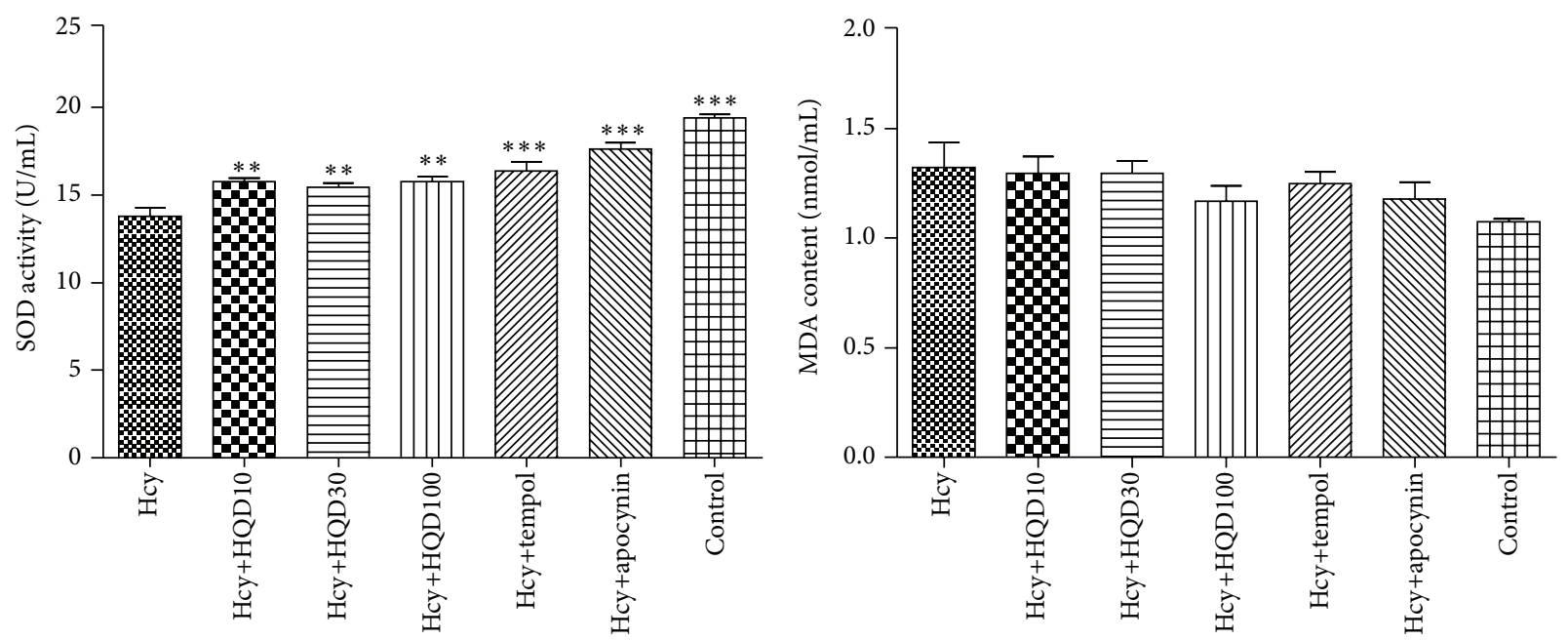

(c)

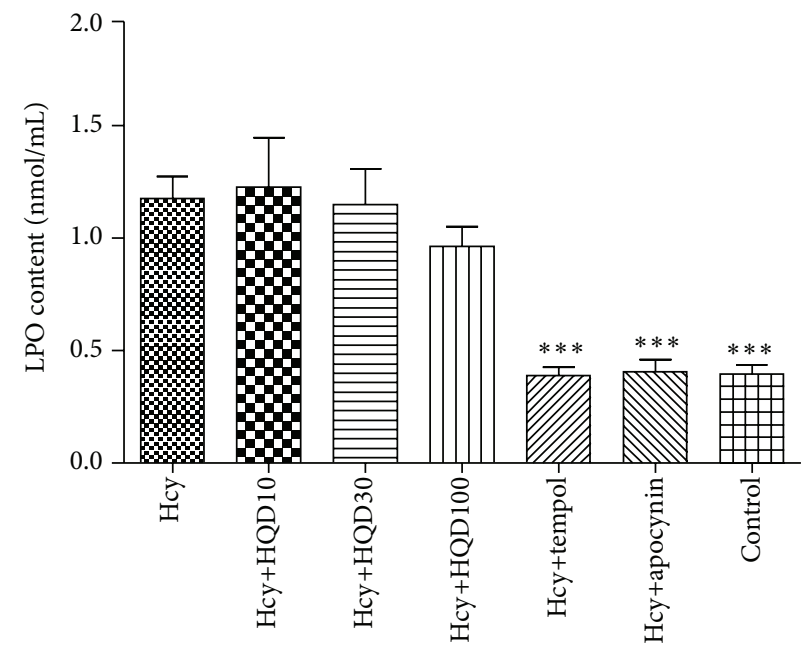

(e)

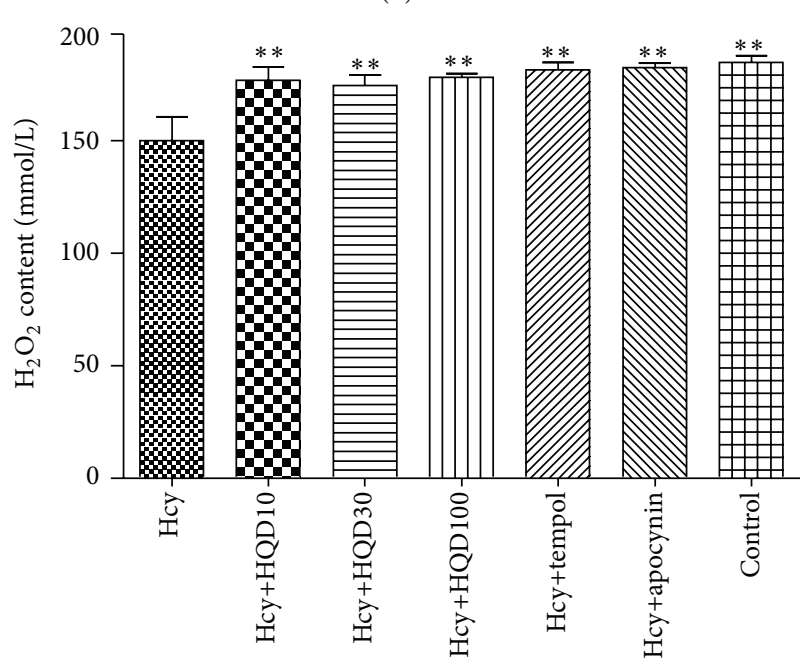

(f)

FIgURE 6: (a) ROS generation was examined by DHE staining. Bar represented $100 \mu \mathrm{m}$. (A) Hcy group; (B) Hcy+HQT10 group; (C) Hcy+HQT30 group; (D) Hcy+HQT100 group; (E) Hcy+tempol group; (F) Hcy+apocynin group; and (G) control group. (b) Relative fluorescence intensity of intracellular ROS. (c) SOD activity. (d) MDA content. (e) LPO content. (f) $\mathrm{H}_{2} \mathrm{O}_{2}$ content. Data are shown as means \pm SEM, $n=3$; HQD: Huang Qi decoction; control: Hcy and HQD untreated group; Hcy: HUVECs treated with Hcy; HQD10: HUVECs group treated with Hcy and HQD at a concentration of $10 \mu \mathrm{g} / \mathrm{mL}$; HQD30: HUVECs group treated with Hcy and HQD at a concentration of $30 \mu \mathrm{g} / \mathrm{mL}$; HQD100: HUVECs group treated with Hcy and HQD at a concentration of $100 \mu \mathrm{g} / \mathrm{mL}$; tempol: HUVECs group treated with Hcy and tempol; and apocynin: HUVECs group treated with Hcy and apocynin. ${ }^{*} P<0.05,{ }^{* *} P<0.01$, and ${ }^{* * *} P<0.001$ versus the Hcy group. 


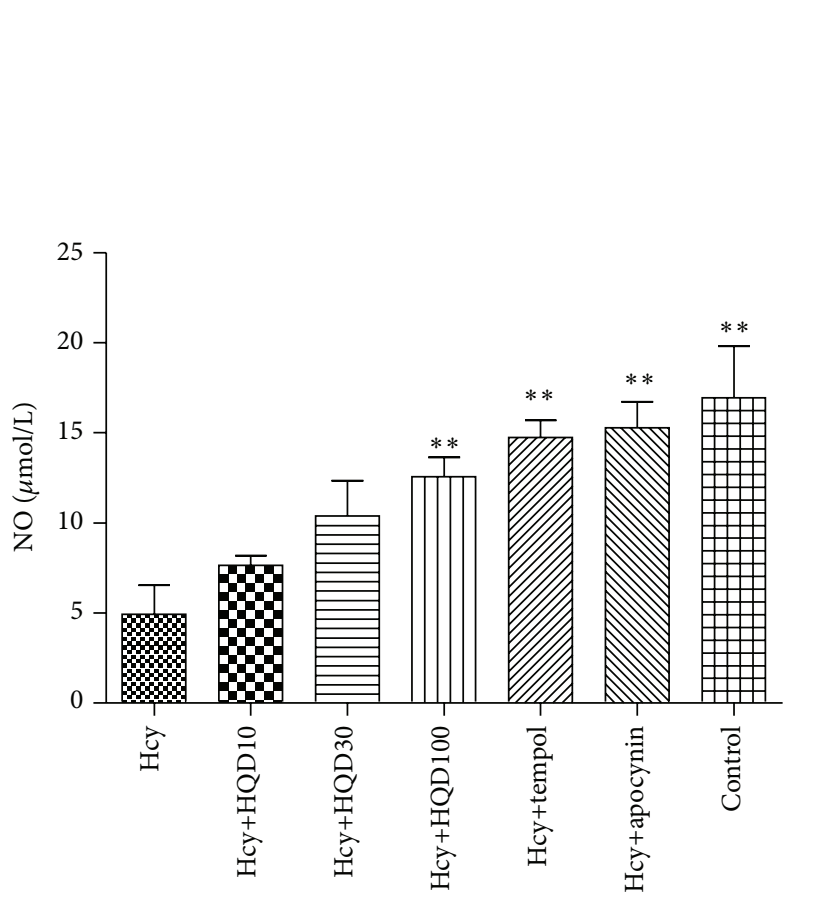

(a)
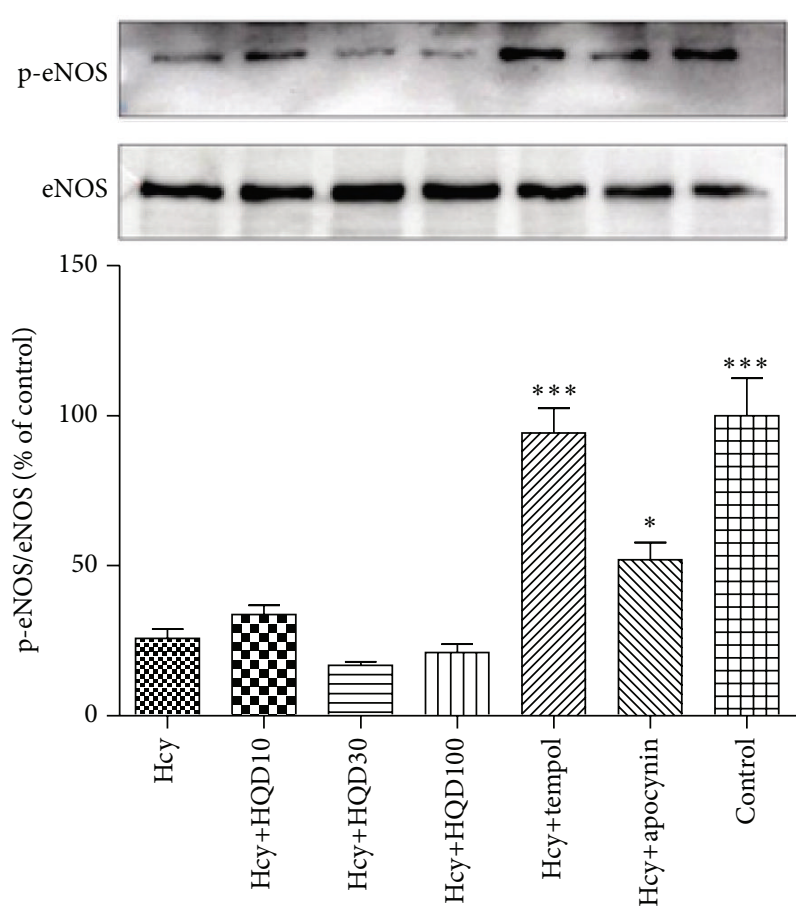

(b)

FIgure 7: (a) NO production. (b) Phosphorylation of eNOS. Data are shown as means \pm SEM, $n=3$; HQD: Huang Qi decoction; control: Hcy and HQD untreated group; Hcy: HUVECs treated with Hcy; HQD10: HUVECs group treated with Hcy and HQD at a concentration of $10 \mu \mathrm{g} / \mathrm{mL}$; HQD30: HUVECs group treated with Hcy and HQD at a concentration of $30 \mu \mathrm{g} / \mathrm{mL}$; HQD100: HUVECs group treated with Hcy and HQD at a concentration of $100 \mu \mathrm{g} / \mathrm{mL}$; tempol: HUVECs group treated with Hcy and tempol; and apocynin: HUVECs group treated with Hcy and apocynin. ${ }^{*} P<0.05,{ }^{* *} P<0.01$, and ${ }^{* * *} P<0.001$ versus the Hcy group.

severe hyperhomocysteinemia $(>100 \mu \mathrm{M})$. Vascular dilation of preconstricted vessels in response to ACh was endothelium dependent. Our results showed that exposure of intact vessels to Hcy remarkably decreased ACh-induced vasorelaxation, implicating that Hcy would cause endothelium damage. To explore the role of ROS, we used tempol, a putative superoxide dismutase (SOD) mimetic which could scavenge superoxide anions. As expected, tempol significantly reversed the increased superoxide anions generation and LPO levels in Hcy group. Additionally, cells were equipped with antioxidant defense including SOD, which could convert superoxide anions to $\mathrm{H}_{2} \mathrm{O}_{2}$. Tempol increased activity of antioxidants SOD which was indicated by elevated levels of $\mathrm{H}_{2} \mathrm{O}_{2}$ compared with Hcy group. Considering these indicative markers of oxidative stress, tempol improved Hcy-induced endothelium-dependent vasodilatory impairment, indicating that ROS might be involved in Hcy-induced endothelium damage. A major source and modulatory enzyme of ROS in the vasculature was NADPH oxidase, a multisubunit enzyme which was composed of membrane-associated cytochrome b558 including p22phox and gp91phox subunits and cytoplasmic subunits including p47phox, p67phox, p40phox, and the small G protein Rac [22]. To exactly explore whether NADPH oxidase derived ROS played a role, apocynin, an NADPH oxidase inhibitor, was applied [23]. Compared with Hcy, apocynin effectively decreased NADPH oxidase expression and ROS production and dilated arterial vessels ex vivo, putatively verifying that NADPH oxidase-dependent ROS generation might exert effects on Hcy-induced impaired vasodilation. Thus antioxidative strategy might improve Hcyinduced endothelial dysfunction.

HQD mainly contained Astragalus (Huang Qi), Poria (Fu Ling), Trichosanthes (Gua Lou), Ophiopogon (Mai Dong), Schisandra (Wu Wei Zi), licorice (Gan Cao), and Rehmannia (Di Huang). These components had been reported to exhibit antioxidant pharmacological properties in various tissues and cells. Accordingly, we suspected that HQD might have potential to ameliorate vascular endothelial dysfunction. In our study, HQD at a dosage larger than $100 \mu \mathrm{g} / \mathrm{mL}$ would induce contraction in PE-precontracted endothelium-intact rings, while $\mathrm{HQD}$ at the concentrations ranging from 10 to $100 \mu \mathrm{g} / \mathrm{mL}$ suppressed the contraction induced by PE in aortic rings with endothelium. Such result was observed in another research in which the treatment with high dose of Huang Qi increased vascular tone [24]. Therefore concentrations of HQD ranging from 10 to $100 \mu \mathrm{g} / \mathrm{mL}$ were applied in our subsequent experiments. As expected, HQD at a proper dosage of 30 or $100 \mu \mathrm{g} / \mathrm{mL}$ significantly reversed the inhibitory effects of Hcy on ACh-induced vasorelaxation. Moreover, HQD at an appropriate dosage significantly restored the increased superoxide anions and decreased SOD induced by Hcy. Meanwhile, HQD at a dosage of $100 \mu \mathrm{g} / \mathrm{mL}$ 


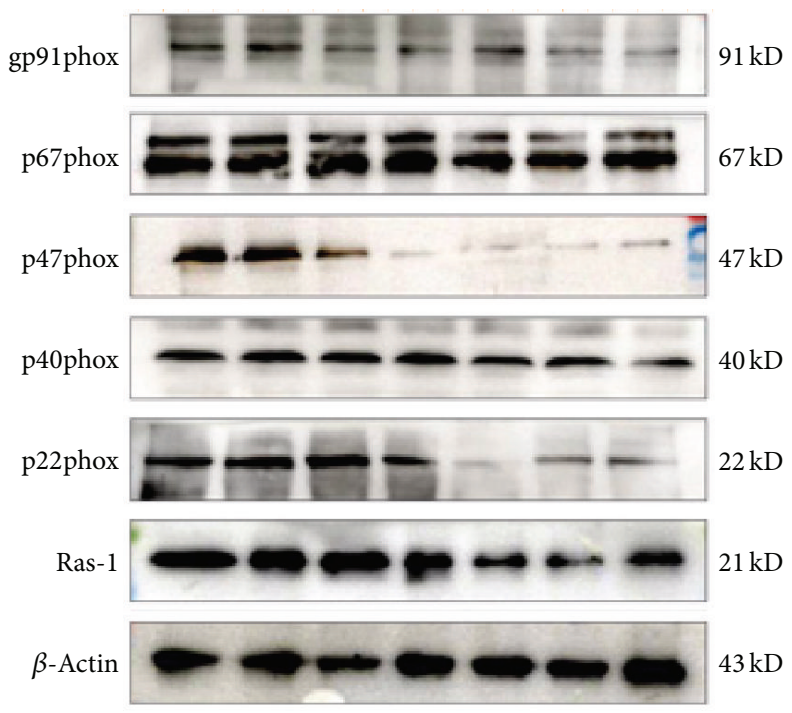

(a)

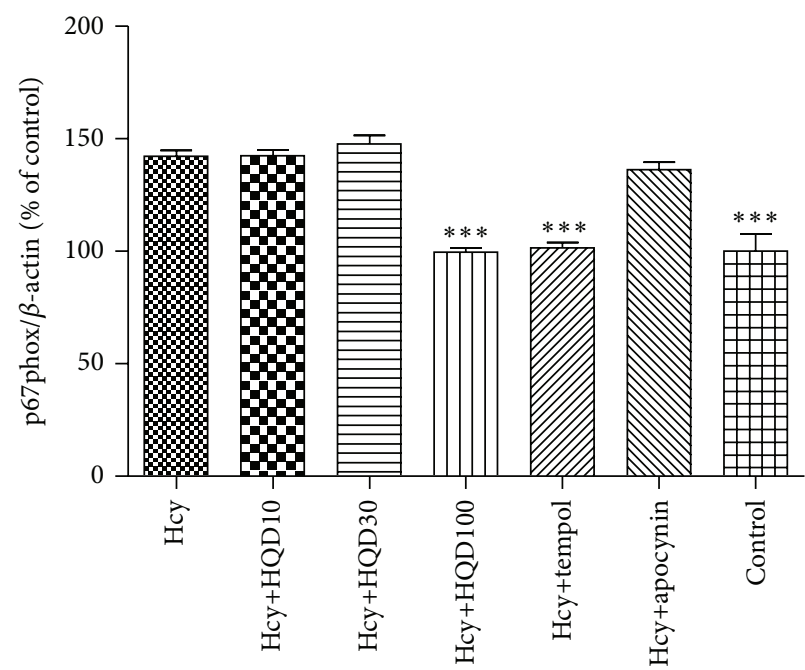

(c)

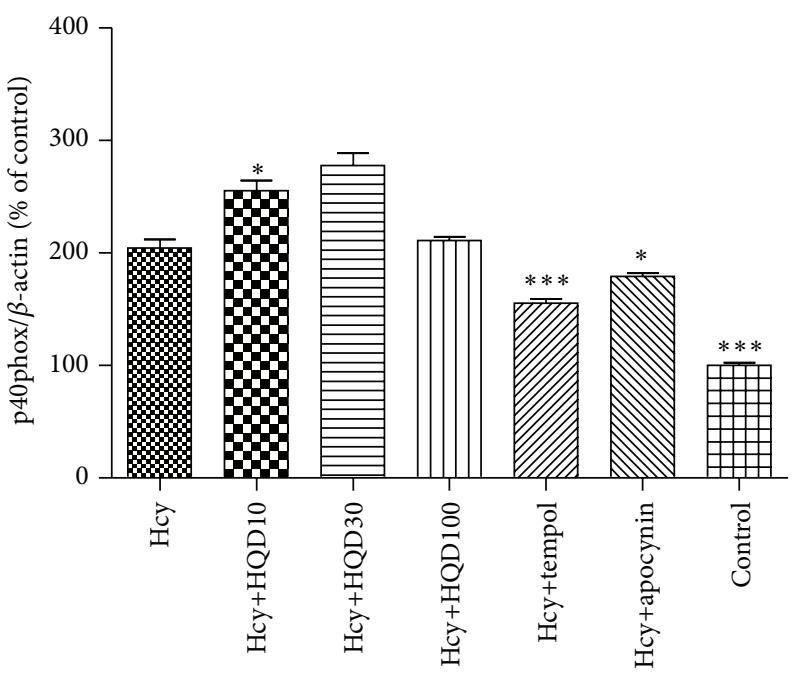

(e)

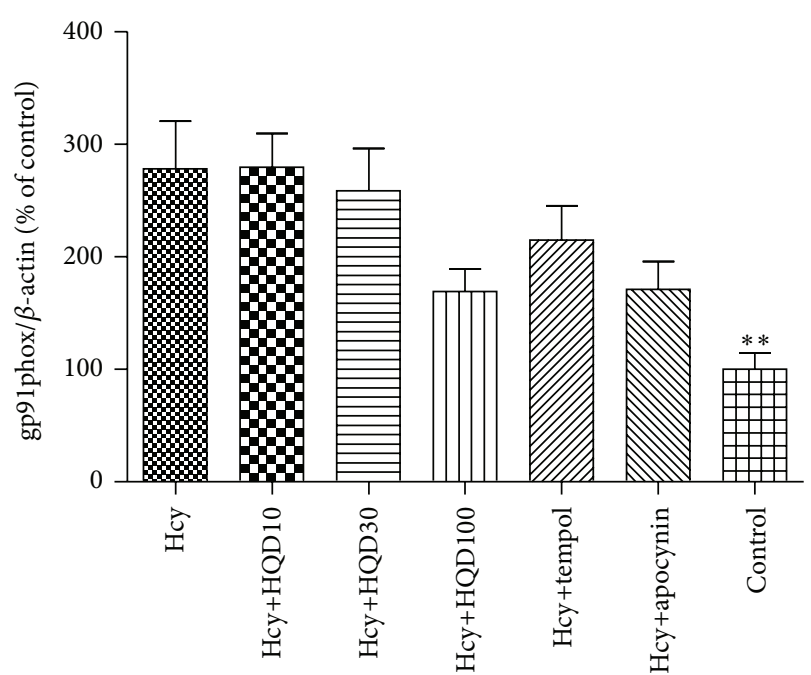

(b)

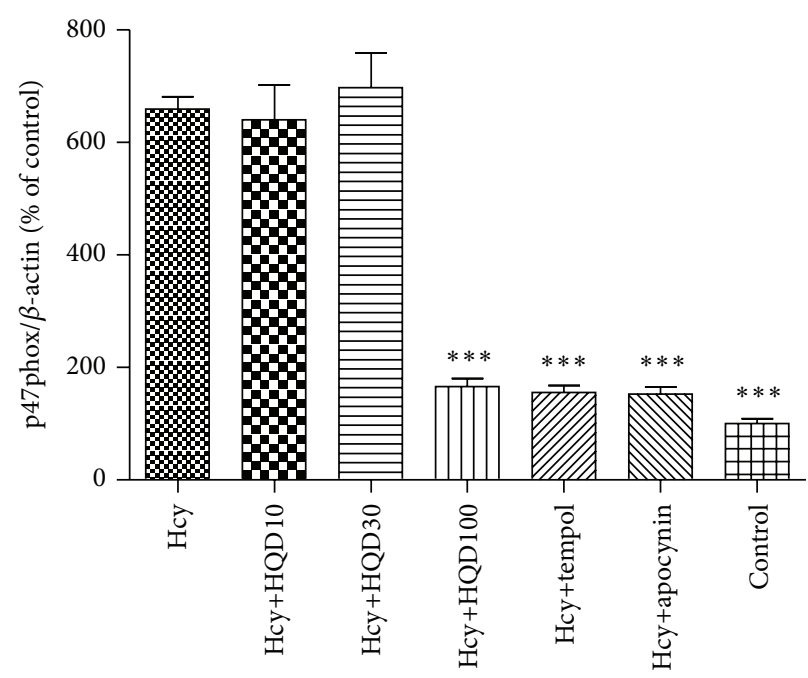

(d)

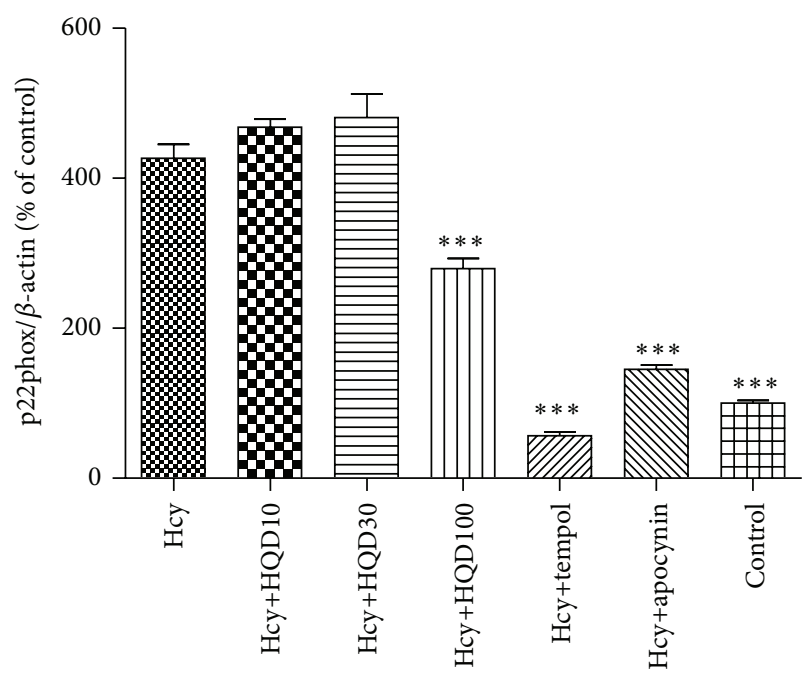

(f)

FIgURE 8: Continued. 


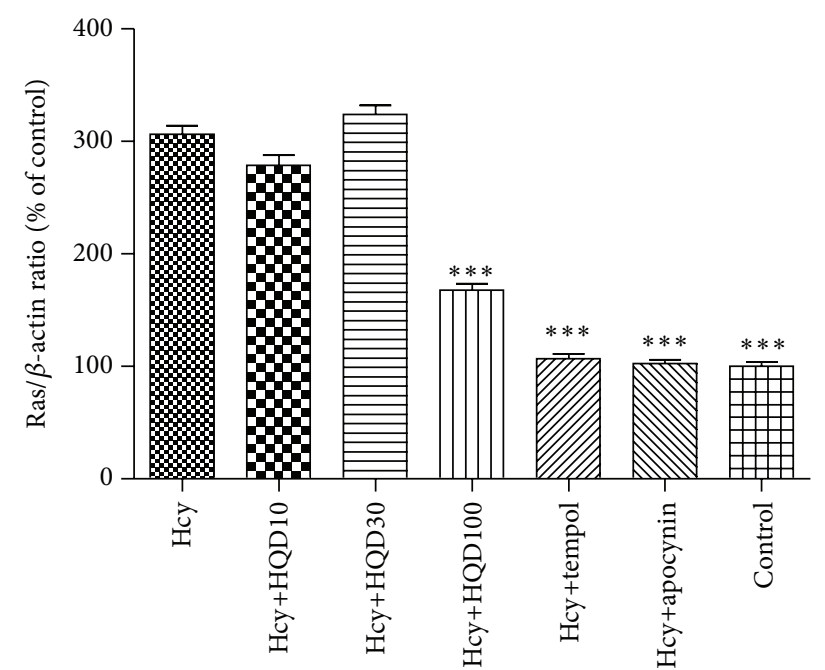

(g)

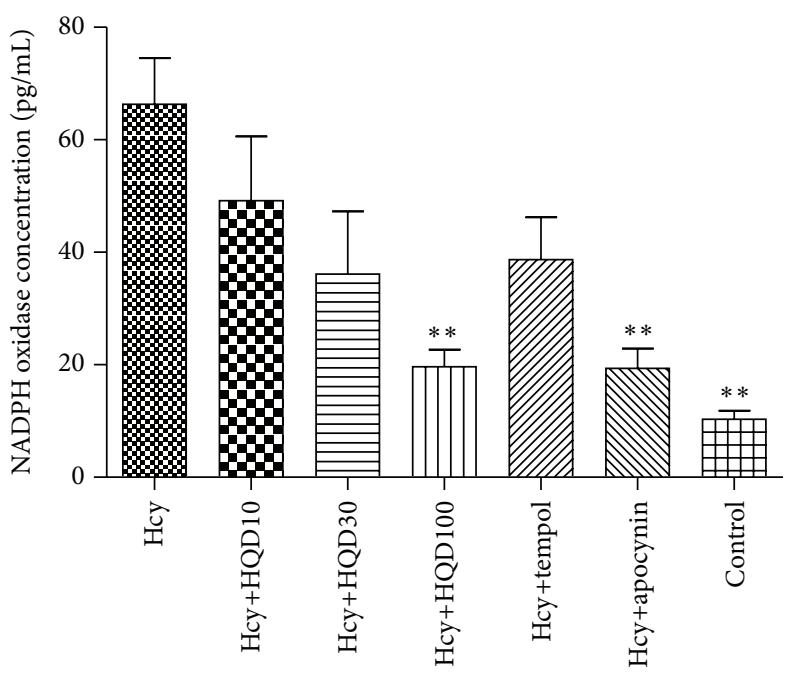

(h)

FIGURE 8: Effects of HQD on the intracellular and extracellular concentration of NADPH oxidase. (a) Western blot for NADPH oxidase subunits including gp91phox, p67phox, p47phox, p40phox, p22phox, and Ras-1. (b)-(g) Statistical analysis of (a). (h) Extracellular concentration of NADPH oxidase. HQD: Huang Qi decoction; normal: Hcy and HQD untreated group; Hcy: HUVECs treated with Hcy; HQD10: HUVECs group treated with Hcy and HQD at a concentration of $10 \mu \mathrm{g} / \mathrm{mL}$; HQD30: HUVECs group treated with Hcy and HQD at a concentration of $30 \mu \mathrm{g} / \mathrm{mL}$; HQD100: HUVECs group treated with Hcy and HQD at a concentration of $100 \mu \mathrm{g} / \mathrm{mL}$; tempol: HUVECs group treated with Hcy and tempol; and apocynin: HUVECs group treated with Hcy and apocynin. ${ }^{*} P<0.05,{ }^{* *} P<0.01$, and ${ }^{* * *} P<0.001$ versus the Hcy group.

decreased the expression of NADPH oxidase, which was one of the sources of ROS. This coordinately demonstrated that there existed an association between the protective effect against Hcy-induced endothelial dysfunction and the inhibitory activity against Hcy-induced increased expression of NADPH oxidase and NADPH oxidase derived ROS. Similar antioxidant activity had been observed in previous reports. In these studies, Astragalus, the component of HQD, was documented to block oxidative stress-induced apoptosis in kidney epithelium [25]. Wojcikowski et al. also demonstrated that 55 kinds of medicinal herbs, including the constituents of HQD such as Astragalus and licorice, had an antioxidant capacity [26]. Therefore, the protective effects of HQD on Hcy-triggered endothelial dysfunction might be attributed to the antioxidative activity of its components. However, the components responsible for the protective performance need to be further explored.

NO was a major regulatory factor of vascular relaxation and endothelial function [27]. In our investigation, an inhibitor of NO synthase, L-NAME, suppressed the improved effects of HQD of $100 \mu \mathrm{g} / \mathrm{mL}$ on Hcy-induced impaired vasorelaxation. Such observation suggested that protective effects of HQD on Hcy-induced vascular endothelial dysfunction were likely to be linked with improved bioavailability of endothelial NO. Actually, Hcy treatment exactly declined NO production as well as the phosphorylation of eNOS which regulated NO generation. HQD at a dose of $100 \mu \mathrm{g} / \mathrm{mL}$ increased production of NO in comparison with Hcy treatment and HQD at the dose 10 or $30 \mu \mathrm{g} / \mathrm{mL}$ showed similar effects to Hcy. Hcy-triggered overgeneration of ROS in the vascular endothelium, which were formed via oxidation of the sulfhydryl base group in Hcy, had been considered to impair bioavailability of endothelium derived NO, resulting in impaired vasodilation [21]. On one hand, ROS could react with $\mathrm{NO}$ to consume $\mathrm{NO}$ and form a kind of deleterious oxidative molecule, which would further cause oxidative injury in endothelial cells. On the other hand, endothelial NO synthase (eNOS) might be oxidized or inhibited from activation, thus negatively influencing the derivation of NO. Both of abovementioned phenomena would contribute to the decreased NO bioavailability and impaired endothelial NO mediated vasodilation. With the antioxidative activity, HQD at an appropriate dosage could alleviate impaired NO bioavailability induced by Hcy, thus improving impaired vasodilation.

An impairment of NO mediated endothelium-dependent relaxation and an enhanced generation of ROS and NADPH oxidase were observed following Hcy exposure. Therefore, regulating the activity of eNOS and production of $\mathrm{NO}$ via controlling the formation of ROS might be a key strategy for attenuation of endothelial dysfunction induced by Hcy. In the current study, HQD could induce production of $\mathrm{NO}$ and decrease ROS through attenuation of ROS-producing enzymes, respectively. Therefore, HQD could alleviate Hcyinduced endothelial dysfunction. However, we have not addressed the ROS deriving from all of vascular oxidase, but focused on ROS production involving NADPH oxidase.

\section{Conclusion}

The current study indicated that HQD exerted a favorable effect on Hcy-induced impaired vasomotor response of aortic 
rings and oxidative stress in HUVECs via partial antioxidant mechanism. Collectively, HQD at a proper dosage provides an alternative for amelioration of oxidative stress-associated endothelial dysfunction, thereby potentially preventing diabetic related vascular disorders.

\section{Competing Interests}

The authors declare that there are no competing interests regarding the publication of this paper.

\section{Acknowledgments}

This work was supported by the Construct Program of the Key Discipline of State Administration of Traditional Chinese Medicine of the People's Republic of China, Leading Academic Discipline Project of State Administration of Traditional Chinese Medicine of China, Talent Project of Integrative Medicine of Shanghai Municipal Health Bureau (ZYSNXD012-RC-ZXY), and Key Medical Discipline Project of Shanghai Municipal Health Bureau (ZK2015A18).

\section{References}

[1] A. Shemyakin, O. Kövamees, A. Rafnsson et al., "Arginase inhibition improves endothelial function in patients with coronary artery disease and type 2 diabetes mellitus," Circulation, vol. 126, no. 25, pp. 2943-2950, 2012.

[2] M. Muñoz, A. Sánchez, M. Pilar Martínez et al., "COX-2 is involved in vascular oxidative stress and endothelial dysfunction of renal interlobar arteries from obese Zucker rats," Free Radical Biology and Medicine, vol. 84, pp. 77-90, 2015.

[3] M. S. El-Awady, D. S. El-Agamy, G. M. Suddek, and M. A. Nader, "Propolis protects against high glucose-induced vascular endothelial dysfunction in isolated rat aorta," Journal of Physiology and Biochemistry, vol. 70, no. 1, pp. 247-254, 2014.

[4] Z. Cheng, X. Jiang, W. D. Kruger et al., "Hyperhomocysteinemia impairs endothelium-derived hyperpolarizing factor-mediated vasorelaxation in transgenic cystathionine beta synthase-deficient Mice," Blood, vol. 118, no. 7, pp. 1998-2006, 2011.

[5] M. Olukman, C. E. Orhan, F. G. Çelenk, and S. Ülker, "Apocynin restores endothelial dysfunction in streptozotocin diabetic rats through regulation of nitric oxide synthase and NADPH oxidase expressions," Journal of Diabetes and Its Complications, vol. 24, no. 6, pp. 415-423, 2010.

[6] Y. Yin, F. Qi, Z. Song, B. Zhang, and J. Teng, "Ferulic acid combined with astragaloside IV protects against vascular endothelial dysfunction in diabetic rats," BioScience Trends, vol. 8, no. 4, pp. 217-226, 2014.

[7] Q. Wang, S. Chen, L. Han et al., "Antioxidant activity of carboxymethyl $(1 \rightarrow 3$ )- $\beta$-d-glucan (from the sclerotium of Poria cocos) sulfate (in vitro)," International Journal of Biological Macromolecules, vol. 69, pp. 229-235, 2014.

[8] B. Perry, J. Zhang, T. Saleh, and Y. Wang, "Liuwei dihuang, a traditional Chinese herbal formula, suppresses chronic inflammation and oxidative stress in obese rats," Journal of Integrative Medicine, vol. 12, no. 5, pp. 447-454, 2014.

[9] W. Li, X.-N. Qu, Y. Han, S.-W. Zheng, J. Wang, and Y.-P. Wang, "Ameliorative effects of 5-hydroxymethyl-2-furfural (5-HMF) from Schisandra chinensis on alcoholic liver oxidative injury in mice," International Journal of Molecular Sciences, vol. 16, no. 2, pp. 2446-2457, 2015.

[10] B. Li, L. Zhu, T. Wu et al., "Effects of triterpenoid from schisandra chinensis on oxidative stress in alcohol-induced liver injury in rats," Cell Biochemistry and Biophysics, vol. 71, no. 2, pp. 803-811, 2015.

[11] S. Zhang, Y. Zhang, H. Li et al., "Antioxidant and anti-excitotoxicity effect of Gualou Guizhi decoction on cerebral ischemia/reperfusion injury in rats," Experimental and Therapeutic Medicine, vol. 9, no. 6, pp. 2121-2126, 2015.

[12] L. Hu, Y. Sun, and J. Hu, "Catalpol inhibits apoptosis in hydrogen peroxide-induced endothelium by activating the PI3K/Akt signaling pathway and modulating expression of Bcl-2 and Bax," European Journal of Pharmacology, vol. 628, no. 1-3, pp. 155-163, 2010.

[13] J. C. Qian, F. R. Jiang, B. Wang et al., "Ophiopogonin D prevents $\mathrm{H}_{2} \mathrm{O}_{2}$-induced injury in primary human umbilical vein endothelial cells," Journal of Ethnopharmacology, vol. 128, no. 2, pp. 438-445, 2010.

[14] T. S. Sun, R. Liu, and Y.-X. Cao, "Vasorelaxant and antihypertensive effects of formononetin through endothelium-dependent and -independent mechanisms," Acta Pharmacologica Sinica, vol. 32, no. 8, pp. 1009-1018, 2011.

[15] M. Ajay, F. I. Achike, A. M. Mustafa, and M. R. Mustafa, "Effect of quercetin on altered vascular reactivity in aortas isolated from streptozotocin-induced diabetic rats," Diabetes Research and Clinical Practice, vol. 73, no. 1, pp. 1-7, 2006.

[16] L. Feng, M.-M. Zhu, M.-H. Zhang et al., "Protection of glycyrrhizic acid against AGEs-induced endothelial dysfunction through inhibiting RAGE/NF- $\kappa$ B pathway activation in human umbilical vein endothelial cells," Journal of Ethnopharmacology, vol. 148, no. 1, pp. 27-36, 2013.

[17] B. K. Nirala, V. Perumal, and N. K. Gohil, "Glycated serum albumin stimulates expression of endothelial cell specific molecule-1 in human umbilical vein endothelial cells: implication in diabetes mediated endothelial dysfunction," Diabetes and Vascular Disease Research, vol. 12, no. 4, pp. 290-297, 2015.

[18] X. Yang, W. Yao, Q. Li et al., "Mechanism of Tang Luo Ning effect on attenuating of oxidative stress in sciatic nerve of STZinduced diabetic rats," Journal of Ethnopharmacology, vol. 174, pp. 1-10, 2015.

[19] A. Vikram, Y.-R. Kim, S. Kumar et al., "Canonical Wnt signaling induces vascular endothelial dysfunction via p66Shc-regulated reactive oxygen species," Arteriosclerosis, Thrombosis, and Vascular Biology, vol. 34, no. 10, pp. 2301-2309, 2014.

[20] N. Tyagi, K. C. Sedoris, M. Steed, A. V. Ovechkin, K. S. Moshal, and S. C. Tyagi, "Mechanisms of homocysteine-induced oxidative stress," American Journal of Physiology-Heart and Circulatory Physiology, vol. 289, no. 6, pp. H2649-H2656, 2005.

[21] C.-S. Kim, Y.-R. Kim, A. Naqvi et al., "Homocysteine promotes human endothelial cell dysfunction via site-specific epigenetic regulation of p66shc," Cardiovascular Research, vol. 92, no. 3, pp. 466-475, 2011.

[22] W. M. T. Kuwabara, L. Zhang, I. Schuiki, R. Curi, A. Volchuk, and T. C. Alba-Loureiro, "NADPH oxidase-dependent production of reactive oxygen species induces endoplasmatic reticulum stress in neutrophil-like HL60 cells," PLOS ONE, vol. 10, no. 2, Article ID e0116410, 2015.

[23] D. A. Rizzetti, J. G. D. Torres, A. G. Escobar et al., "Apocynin prevents vascular effects caused by chronic exposure to low concentrations of mercury," PLoS ONE, vol. 8, no. 2, article e55806, 2013. 
[24] B.-Q. Zhang, S.-J. Hu, L.-H. Qiu et al., "Diphasic effects of Astragalus membranaceus Bunge (Leguminosae) on vascular tone in rat thoracic aorta," Biological \& Pharmaceutical Bulletin, vol. 28, no. 8, pp. 1450-1454, 2005.

[25] M. Shahzad, D. M. Small, C. Morais, K. Wojcikowski, A. Shabbir, and G. C. Gobe, "Protection against oxidative stressinduced apoptosis in kidney epithelium by Angelica and Astragalus," Journal of Ethnopharmacology, vol. 179, pp. 412-419, 2016.

[26] K. Wojcikowski, L. Stevenson, D. Leach, H. Wohlmuth, and G. Gobe, "Antioxidant capacity of 55 medicinal herbs traditionally used to treat the urinary system: a comparison using a sequential three-solvent extraction process," The Journal of Alternative and Complementary Medicine, vol. 13, no. 1, pp. 103-109, 2007.

[27] A. Magenta, S. Greco, M. C. Capogrossi, C. Gaetano, and F. Martelli, "Nitric oxide, oxidative stress, and $\mathrm{p} 66^{\text {Shc }}$ interplay in diabetic endothelial dysfunction," BioMed Research International, vol. 2014, Article ID 193095, 16 pages, 2014. 


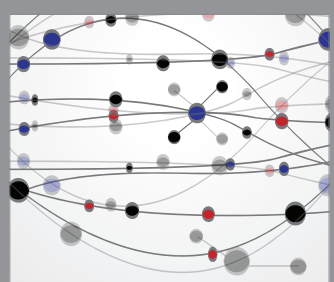

The Scientific World Journal
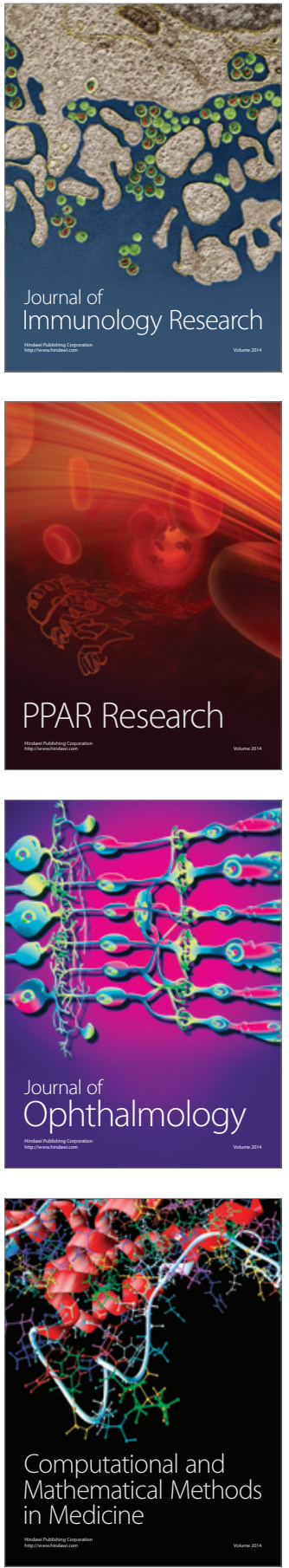

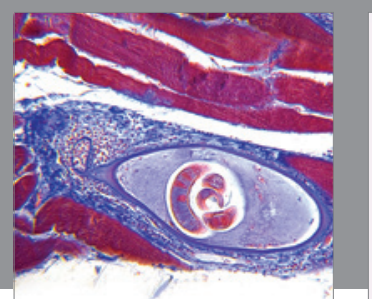

Gastroenterology Research and Practice

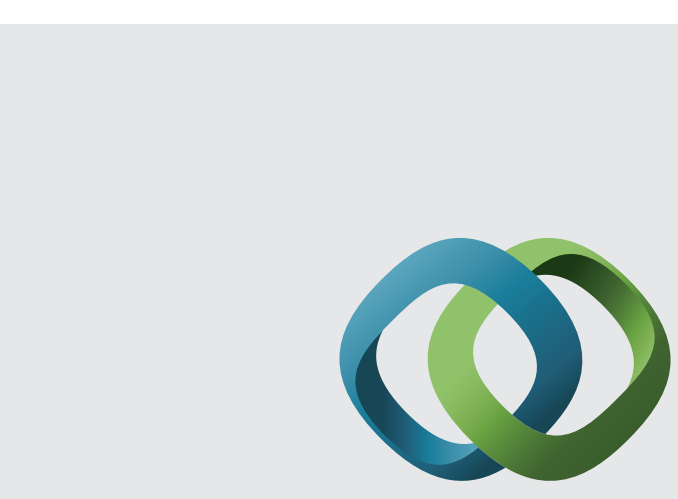

\section{Hindawi}

Submit your manuscripts at

http://www.hindawi.com
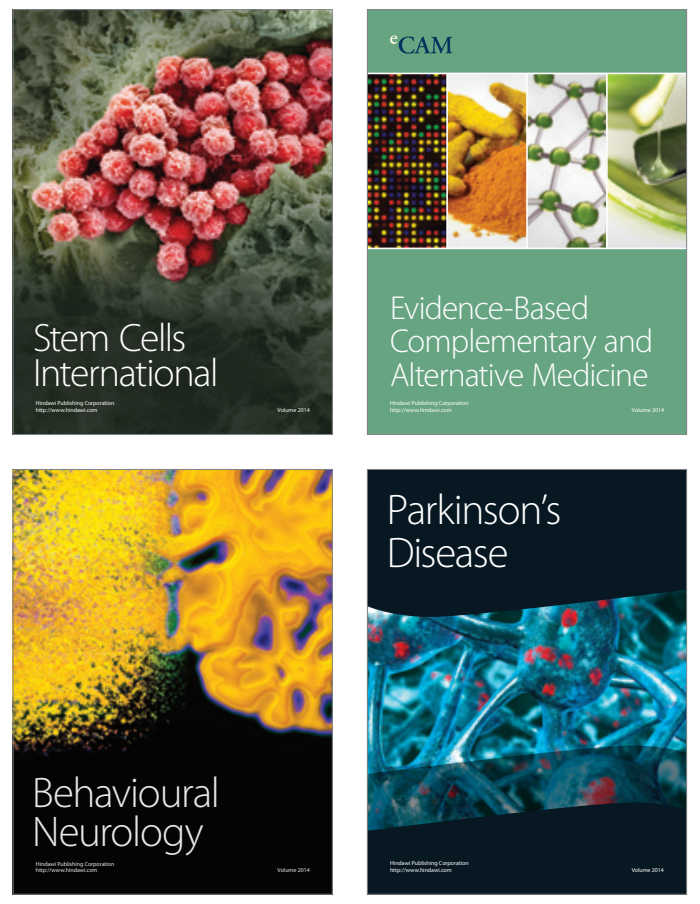
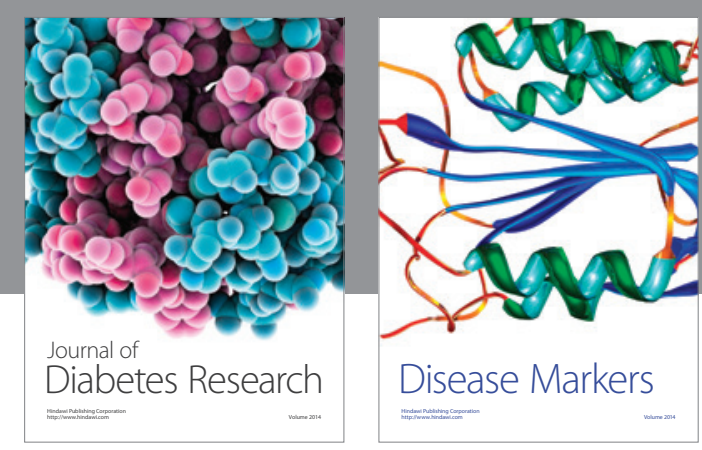

Disease Markers
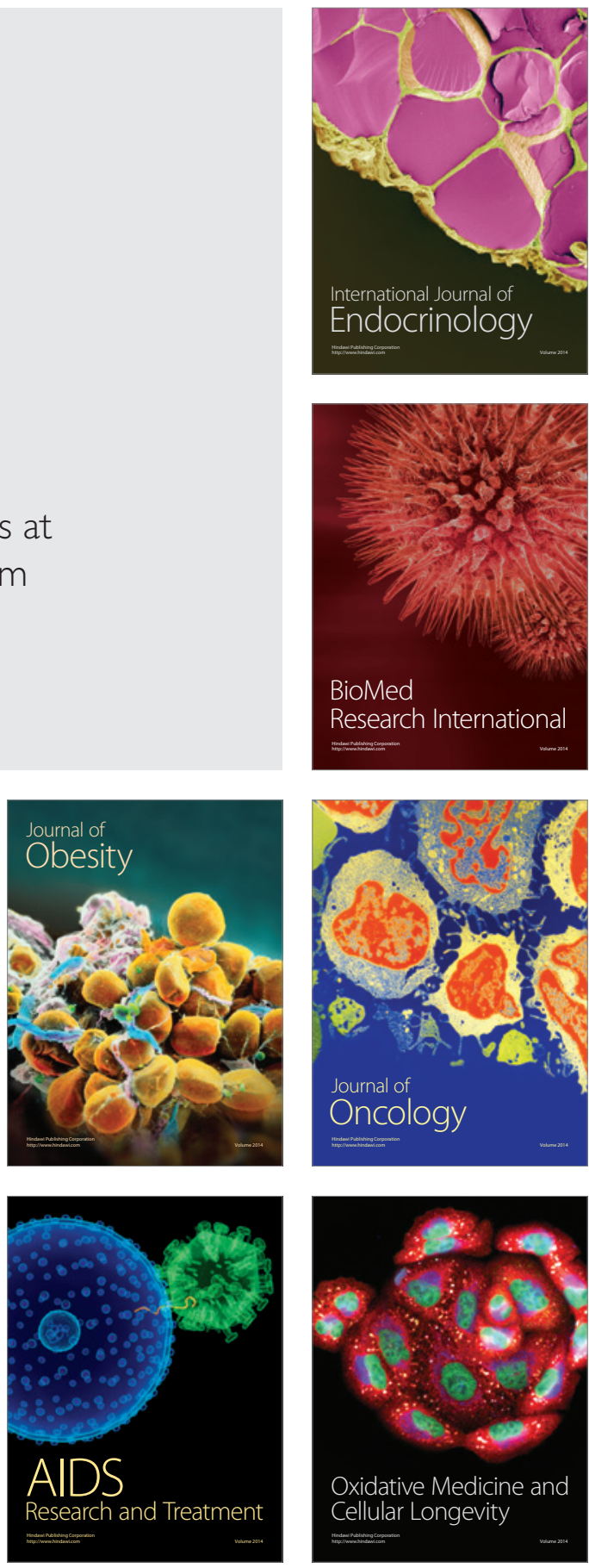